DIAS-STP-93-14

August 1993

\title{
On the Emergence of Gauge Structures and Generalized Spin when Quantizing on a Coset Space
}

\author{
David McMullan* and Izumi Tsutsui \\ Dublin Institute for Advanced Studies \\ 10 Burlington Road \\ Dublin 4 \\ Ireland \\ e-mail:mcmullan@stp.dias.ie tsutsui@stp.dias.ie
}

\begin{abstract}
It has been known for some time that there are many inequivalent quantizations possible when the configuration space of a system is a coset space $\mathrm{G} / \mathrm{H}$. Viewing this classical system as a constrained system on the group $\mathrm{G}$, we show that these inequivalent quantizations can be recovered from a generalization of Dirac's approach to the quantization of such a constrained system within which the classical first class constraints (generating the $\mathrm{H}$-action on $\mathrm{G}$ ) are allowed to become anomalous (second class) when quantizing. The resulting quantum theories are characterized by the emergence of a Yang-Mills connection, with quantized couplings, and new 'spin' degrees of freedom. Various applications of this procedure are presented in detail: including a new account of how spin can be described within a pathintegral formalism, and how on $S^{4}$ chiral spin degrees of freedom emerge, coupled to a BPST instanton.
\end{abstract}

* Address after 1.9.93: School of Mathematics and Statistics, University of Plymouth, Drake Circus, Plymouth, Devon PL4 8AA, U.K. 


\section{Introduction}

Yang-Mills theory provides the theoretical basis for all descriptions of high energy physics, yet we still know very little about the actual quantization of such a system. One of the biggest problems is that the very gauge invariance that makes the theory so attractive implies that not all components of the gauge field are physical. This is a familiar problem in electrodynamics where it is only the transverse components of the photon that have an independent physical significance. Based on this observation one can quantize electrodynamics after reducing it to the true degrees of freedom ${ }^{1}$. The technical complication in Yang-Mills theory then being that there is no similar (or at least useful) classical reduction to the true degrees of freedom, and hence we are forced to quantize more than the physical content of the theory. Clearly, some method is then needed for extracting the physical sector from the extended quantum theory.

A systematic approach to this type of problem was initiated by Dirac [1] and has become known as Dirac's approach to the quantization of a constrained system. Working in a slightly more general context than the specific example of Yang-Mills theory (for the traditional quantization of Yang-Mills theory we refer to Ref. 2), consider a classical system given by an extended phase space with first class constraints,

$$
\phi_{i}=0
$$

Being first class simply means that under the Poisson bracket the constraints close, i.e.,

$$
\left\{\phi_{i}, \phi_{j}\right\}=f_{i j}^{k} \phi_{k}
$$

for some structure functions $f_{i j}^{k}$. In Yang-Mills theory it is Gauss' law that plays the role of the constraints and the structure functions are actually constants.

The idea behind Dirac's approach to the quantization of such a constrained system is to first quantize on the extended system, ignoring the constraints, then reduce to the physical states by imposing the conditions implementing the constraints (1.1) at the quantum level:

$$
\widehat{\phi}_{i}\left|\psi_{\text {phy }}\right\rangle=0
$$

1 This is, of course, possible only if one is willing to give up the manifest Lorentz invariance of the quantum theory. If not, then even in electrodynamics one has to deal with an extended system in one way or another. 


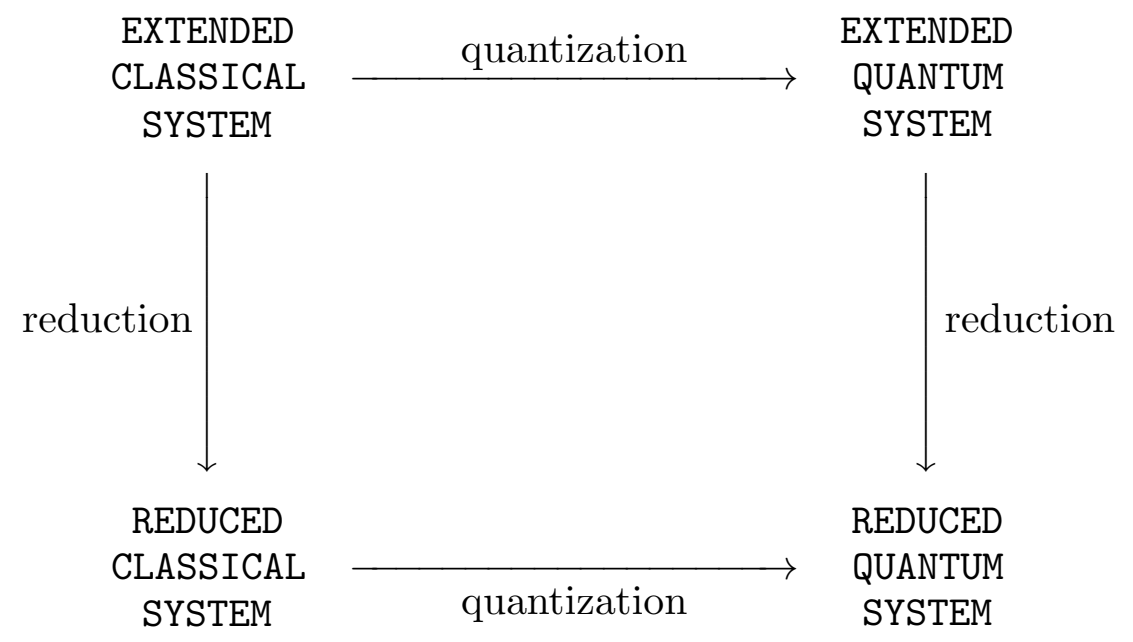

\section{Fig. 1: Traditional point of view on reduction/quantization}

As long as the first class nature (1.2) of the constraints has been preserved (and modulo ordering problems if the structure functions are not constants), condition (1.3) is consistent and should result in a reasonable set of physical states. In fact, it is expected that this process will recover the same set of states arrived at by directly quantizing on the true degrees of freedom. So we can summarize Dirac's approach to the quantization of a constrained system by the (commutative) diagramme in Fig. 1. This freedom of changing the ordering of quantizing and reducing is directly testable in electrodynamics and, although one can find toy models where curvature effects can spoil the commutivity of this description [3], more modern accounts which use ghost variables [4] show that we are able in general to recover the expected quantization on the reduced space from this type of constrained quantization on the extended system. But what is the expected quantization?

Our expectations for what is the correct quantization on the reduced system is based, to a large extent, on our experience with quantizing simple quantum mechanical systems such as the harmonic oscillator or the Hydrogen atom - systems which have very simple configuration spaces $Q$ of the form $\mathbb{R}^{n}$, for some $n$. For electrodynamics the physical configuration space is essentially a linear space, so we are quite confident in our intuition for what is the expected quantum theory. But in Yang-Mills theory the physical configuration space $Q$ is a very complicated space [5], having features more in common with the coset spaces of the form $\mathrm{G} / \mathrm{H}$, for some groups $\mathrm{G}$ and $\mathrm{H}$, rather than $\mathbb{R}^{n}$, for any $n$. 
Quantum theory on a coset space $\mathrm{G} / \mathrm{H}$ is much richer than that on $\mathbb{R}^{n}$. Indeed, it was shown by Mackey [6] that there are many different quantizations possible on $\mathrm{G} / \mathrm{H}$, labelled by the irreducible unitary representations of the group H. After Mackey's work of more than a quarter of a century ago, there appeared a number of other approaches to the quantization on the coset $\mathrm{G} / \mathrm{H}$ (or, more generally, to the quantization of a classical system whose configuration space $Q$ is more complicated than $\mathbb{R}^{n}$ ). Among them are geometric quantization [7], the canonical group approach [8] and a $\mathrm{C}^{*}$-algebric reformulation of Mackey's analysis [9, 10], all of which emphasize different structures and do not appear to be equivalent. Nonetheless, for the coset space $\mathrm{G} / \mathrm{H}$ that we are considering, they all conclude that inequivalent quantizations do exist, and that these different quantum sectors always include those described by Mackey; in this sense we may regard Mackey's approach as the most fundamental one. Recently, a significant advance in understanding the dynamical consequences of these inequivalent quantizations was taken by Landsman and Linden [10], who showed that the different quantum sectors come equipped with a specific type of Yang-Mills field, called the H-connection.

Most of these approaches, including Mackey's, quantize directly on $\mathrm{G} / \mathrm{H}$ by generalizing the canonical commutation relations, and share a common feature that they deal with vector-valued wave functions, rather than the usual scalar-valued ones. As such they suffer severely from techincal difficulties, which hamper the extention to field theory that we are aiming at. In [10] an attempt was made at directly developing a path-integral account of the various quantum sectors by starting with the vector-valued wave functions. Again, the problem is that, due to the vector-valued nature of the states, the path-integral must always be path-ordered, resulting in a 'discrete' path-integral leading to a transition matrix rather than an amplitude. Consequently, one cannot recover the usual expression for the path-integral, that is, as a summation over 'continuous' paths weighted by the exponential of a classical action. That Mackey's original formulation might not be the most natural can also be argued from the fact that the role of the H-connection only became apparent twenty two years after Mackey's book on the subject was published!

The above discussions represent the dual themes of this paper: how should Dirac's approach be extended to take into account the possibility that there are many possible quantizations on the reduced system, and what is the most natural (useful) formulation of Mackey's analysis of the quantum theories on $\mathrm{G} / \mathrm{H}$ ? What we shall find is that these two questions are, in fact, equivalent. That is, in this paper we reformulate Mackey's 
description of quantization so that the use of vector-valued wave functions can be avoided, thus allowing for the construction of a conventional continuous path-integral description of the different quantum sectors. This reformulation will be seen to simplify the quantization significantly, and clarify many of the difficult aspects of Mackey's account of quantizing on $\mathrm{G} / \mathrm{H}$. For instance, after our reformulation the emergence of the H-connection and the gauge structure associated with it - which we feel is central to the physical understanding of the different quantum sectors and to the extention to field theory - can be recognized almost immediately. Furthermore, the vector-valuedness that caused the trouble is converted to a set of observables, called 'generalized spin', that have an effective classical counterpart. For our reformulation we shall adopt a constrained point of view, that is, we shall view the system on $\mathrm{G} / \mathrm{H}$ as a reduction of a larger, albeit simpler to quantize, system. We will then find that, in order to recover Mackey's description of the possible quantizations, a generalized version of Dirac's approach is needed. To our pleasant surprize, the generalization we need turns out to be quite simple: one just replaces the physical state conditions (1.3) with

$$
\widehat{\phi}_{i}\left|\psi_{\text {phy }}\right\rangle=K_{i}\left|\psi_{\text {phy }}\right\rangle
$$

where the $K_{i}$ are constants (integers) characterizing the irreducible unitary representations of the group $\mathrm{H}$.

The conditions (1.4) show that upon quantization the constraints become anomalous (i.e., some of them become second class) and should be treated as such. This result obtained for $Q \simeq \mathrm{G} / \mathrm{H}$ leads us to the following conjecture for the quantization on a generic configuration space $Q$ : one should, in general, expect many inequivalent quantizations on $Q$ if it is 'non-trivial'; these quantizations can be obtained by the generalized Dirac approach, that is, first view the system as a reduction from a 'trivial' one, whose quantization is simple, and then reduce it at the quantum level, taking into account the allowed anomalous nature of the constraints. Accordingly, the previous diagramme, Fig. 1, is no longer appropriate for this constrained approach to quantizating with a generic reduced configuration space $Q$, and needs to be replaced by the diagramme in Fig. 2. Of course, for this generalization to work a number of problems should be solved; for instance, we must find a suitable extended configuration space which is trivial in the above sense, and specify what the anomalous $K_{i}$ are. For the case $Q \simeq \mathrm{G} / \mathrm{H}$ our natural choice for the extended configuration space will be the group $\mathrm{G}$, and it will be shown in this paper that the $K_{i}$ are actually determined correctly from a consistency of the path-integral. Interestingly, this 


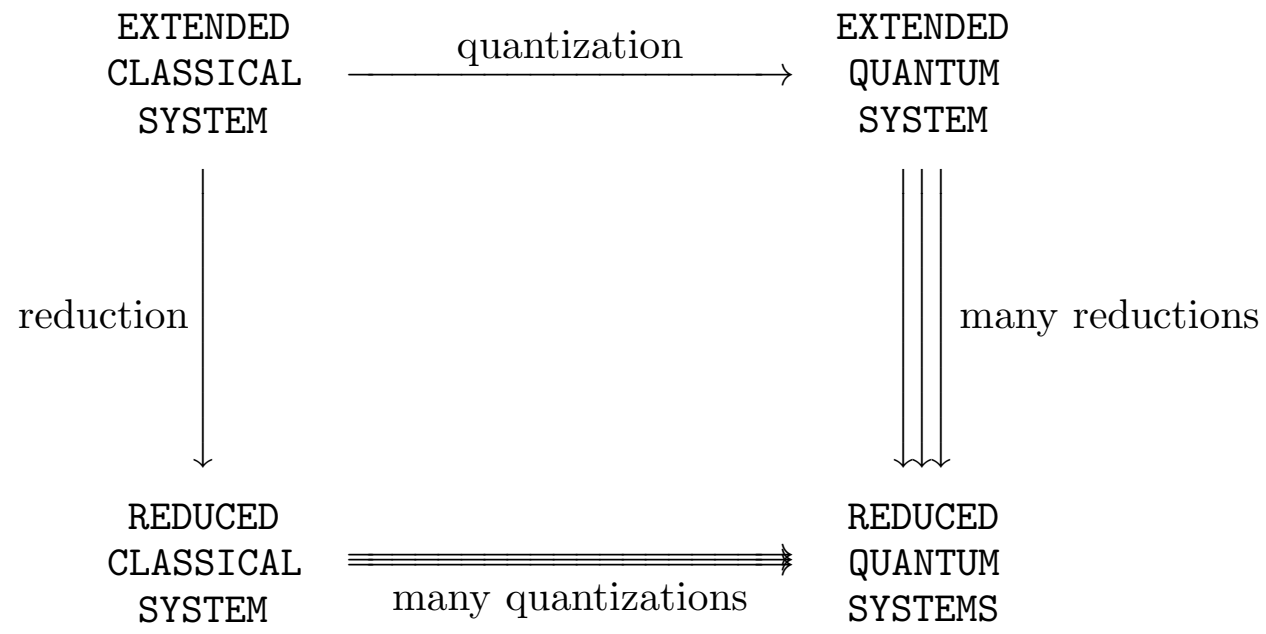

\section{Fig. 2: Generalized version of Dirac's approach}

generalized Dirac approach can be applied to Yang-Mills theory (where again there is a natural choice for the extended configuration space) using the conditions (1.4) with fielddependent $K_{i}$, which yields additional topological terms in the Yang-Mills Lagrangian as a consequence of the inequivalent quantizations [11]. We also mention that this change in the nature of the constraints is familiar when quantizing anomalous gauge theories (see [12] and also the discussion in [2]), which indicates a possible new response to gauge anomalies based on the possibility of exploiting the existence of inequivalent quantizations. In this paper, however, to establish a firm ground for those extentions, and to examine the physical implications of inequivalent quantizations as clearly as possible, we shall confine our arena to the coset spaces $Q \simeq \mathrm{G} / \mathrm{H}$, which is also worth investigating in its own right.

The plan of this paper is as follows. After this introduction, in Sect. 2, Mackey's quantization on $\mathrm{G} / \mathrm{H}$ will be described along with some discussion of how it relates to other direct approaches and some of the problems with its usual description. The aim of this somewhat lengthy review part is to provide a self-contained account of Mackey's formulation, which is later used to prove the equivalence to our (generalized Dirac) approach. In Sect. 3 we illustrate the idea of our reformulation of Mackey's inequivalent quantization by a simple example, where the emergence of spin is explained from particle dynamics on $\mathbb{R}^{3}$. This will end up with a derivation of the path-integral description of spin, proposed by Nielsen and Rohrlich [13], from Mackey's account of quantization applied to the coset space $\widetilde{\mathrm{E}(3)} / \mathrm{SU}(2) \simeq \mathbb{R}^{3}$. After this illustration, in Sect. 4 we present for the general coset space 
a precise definition of our generalization of Dirac's approach, and prove its equivalence with Mackey's account of quantizing on such coset spaces. In Sect. 5 we will develop the path-integral version of our approach presented in Sect. 4, in which the effects of inequivalent quantizations - the H-connection and generalized spin - become transparent. This section can almost be read independently of what has gone before (although Sect. 4.1 is needed for the notation), and we would suggest to the reader that if one is only interested in the final results of our analysis and the applications, then go directly to Sect. 5 to see how simple our methods are. Sect. 6 is devoted to a study of the physical implications of inequivalent quantizations by examining in detail the case $S^{n} \simeq \mathrm{SO}(\mathrm{n}+1) / \mathrm{SO}(\mathrm{n})$ for $n=2,3,4$. The physical interpretation of spin will be given for $S^{3}$ and $S^{4}$, and, in particular on $S^{4}$, it will be shown that chiral spin degrees of freedom couple to a BPST instanton (and anti-instanton), which is the H-connection in this case. Finally, in Sect.7, we shall conclude this analysis with some speculations on the possible ramifications of the ideas presented in this paper. There are also four appendices containing some technical results used in the main text, the most important of which is Appendix A which, among other things, gives our notational conventions for dealing with the many spaces needed in this account of quantization. 


\section{Direct approaches to quantizing on coset spaces}

In this section the quantum mechanics of a particle moving on a coset space $\mathrm{G} / \mathrm{H}$ is reviewed. Among the many approaches that quantize directly on $\mathrm{G} / \mathrm{H}$, we discuss primarily Mackey's, which is the first and the most fundamental one in the sense mentioned earlier. Special emphasis will be placed on the emergence of inequivalent quantizations, labelled by the irreducible unitary representations of the group $\mathrm{H}$, and the effect this has on the dynamics in the different sectors. Later, we shall briefly discuss other approaches together with problems that arise with the applications and extensions of these results.

\subsection{Mackey's approach: the system of imprimitivity}

A spinless particle moving on the flat configuration space $\mathbb{R}^{3}$ provides the most familiar example of the quantization process. The basic observables of this system are those of position $\hat{q}^{\alpha}$ and momentum $\hat{p}_{\alpha}(\alpha=1,2,3)$. These self-adjoint operators satisfy the canonical commutation relations,

$$
\left[\hat{q}^{\alpha}, \hat{q}^{\beta}\right]=0, \quad\left[\hat{p}_{\alpha}, \hat{p}_{\beta}\right]=0 \quad \text { and } \quad\left[\hat{q}^{\alpha}, \hat{p}_{\beta}\right]=i \delta_{\beta}^{\alpha},
$$

which reflects the classical Poisson bracket relations between position and momentum.

To discuss the representations of these commutator relations, and to address the question of their uniqueness, it is best to work with the bounded unitary operators $U(a):=$ $\exp (-i a \cdot \hat{p})$ and $V(b):=\exp (i b \cdot \hat{q})$ where $a, b \in \mathbb{R}^{3}$, in terms of which the canonical commutation relations (2.1) become the Weyl relations:

$$
U(a+b)=U(a) U(b), \quad V(a+b)=V(a) V(b)
$$

and

$$
U(a) V(b) U^{-1}(a)=e^{-i a \cdot b} V(b) .
$$

A particular representation of these is furnished by the Schrödinger representation in which the quantum states are identified with the space $L^{2}\left(\mathbb{R}^{3}\right)$ of wave functions $\psi(q)$ on the configuration space, and the unitary operators $U(a)$ and $V(b)$ become

$$
(U(a) \psi)(q)=\psi(q-a), \quad(V(b) \psi)(q)=e^{i b \cdot q} \psi(q) .
$$


In this representation the position observables $\hat{q}^{\alpha}$ acts on these states as the multiplication operators, while the momentum observables $\hat{p}_{\alpha}$ become the derivative operators $-i \partial / \partial q^{\alpha}$. The Stone-von Neumann theorem then states that all other irreducible unitary representations of the Weyl relations, (2.2) and (2.3), are unitarily equivalent to the Schrödinger representation $(2.4)$.

There are, however, various unsatisfactory aspects to this description of quantization - problems which become serious when we attempt to generalize this construction to more interesting systems. The most obvious problem is that, for a general system whose configuration space is 'non-trivial', we do not expect there to be globally well defined observables satisfying the canonical commutation relations (2.1). Another problem is that we seem to be imposing on the quantum theory the restriction that the observables be constructed solely out of the position and momentum variables, and thereby excluding the possibility of spin (or even other, more exotic, observables). What is surprising, then, is that both of these problems can be resolved, for a wide class of theories, by simply reinterpreting the Weyl relations, (2.2) and (2.3).

To understand this reinterpretation let us recall that in (Dirac's account of) the Schrödinger representation the abstract quantum states $|\psi\rangle$ are expanded in terms of the position eigenstates $|q\rangle: \hat{q}^{\alpha}|q\rangle=q^{\alpha}|q\rangle$, and the wave functions are identified with the components of the state in this position basis, $\psi(q)=\langle q \mid \psi\rangle$. The translation group $\mathrm{G}=\mathbb{R}^{3}$ acts transitively on the configuration space $\mathbb{R}^{3}$ with an action $q \mapsto g q:=q+g$ (we use here $g$ instead of $a$ to stress its group property). The unitary operator $U(g)$ is then defined by

$$
U(g)|q\rangle=|g q\rangle
$$

Acting on the wave functions this becomes

$$
(U(g) \psi)(q)=\langle q|U(g)| \psi\rangle=\left\langle g^{-1} q \mid \psi\right\rangle=\psi\left(g^{-1} q\right)
$$

If $F(q)$ is any bounded function on the configuration space $\mathbb{R}^{3}$ then the spectral theorem asserts that $\widehat{F}(q):=F(\hat{q})$, the operator corresponding to it, can be written as

$$
\widehat{F}(q)=\int_{\mathbb{R}^{3}} d^{3} q F(q)|q\rangle\langle q|,
$$

where the formal expression, $d^{3} q|q\rangle\langle q|$, is to be identified with the spectral measure associated with the self-adjoint operator $\hat{q}$. Using (2.5) and the translation invariance of the measure on $\mathbb{R}^{3}$, we see that

$$
U(g) \widehat{F}(q) U^{-1}(g)=\widehat{F}\left(g^{-1} q\right) .
$$


A unitary representation $U(g)$ of the Lie group $G=\mathbb{R}^{3}$ satisfying the relation (2.8), where $F$ is any bounded function on $\mathbb{R}^{3}$, was called by Mackey ${ }^{2}$ a system of imprimitivity based on $\mathbb{R}^{3}[6]$ (see also [14]). For this abelian group acting transitively on the configuration space Mackey showed that there is a unique representation of this system of imprimitivity, which is just the Schrödinger representation presented above. This reformulation of the Stone-von Neumann theorem by Mackey has allowed us to replace the specific bounded operator $V$ which satisfies the relation (2.3) (the existence of which is closely related to the geometry of $\mathbb{R}^{3}$ ) by an arbitary bounded function $F$ whose operator $\widehat{F}$ satisfies the imprimitivity relation $(2.8)$.

This construction has an immediate generalization. Consider a configuration space on which a group $\mathrm{G}$ acts transitively ${ }^{3}$. If a closed subgroup $\mathrm{H} \subset \mathrm{G}$ is the isotropy group of the action then we can identify the configuration space with the coset space $\mathrm{G} / \mathrm{H}$. We call a unitary representation $U(g)$ of $\mathrm{G}$ a system of imprimitivity based on $\mathrm{G} / \mathrm{H}$ if it satisfies the relation (2.8) for any bounded function $F(q)$ on $\mathrm{G} / \mathrm{H}$.

For example, the translation group $\mathbb{R}$ does not act transitively on the configuration space $\mathbb{R}^{+}$(the positive real line), rather an affine action is needed: $q \rightarrow \lambda q$, for $\lambda \in \mathbb{R}^{+}$. Thus, although operators satisfying the Weyl relations (2.2) and (2.3) cannot be found for $\mathbb{R}^{+}$, a system of imprimitivity can be constructed using this affine action [8]. A less trivial example follows from the simple observation that the Euclidean group $\mathrm{E}(3)=\mathrm{SO}(3) \ltimes \mathbb{R}^{3}$ also acts transitively on $\mathbb{R}^{3}$; so if we wish to study quantum systems invariant not just under translations but under this larger group then we should start with the system of imprimitivity based on $\mathbb{R}^{3}$, now viewed as the coset space $\mathrm{E}(3) / \mathrm{SO}(3)$. This example serves to highlight the basic fact that there is no unique system of imprimitivity to be assigned

2 Mackey's formulation of a system of imprimitivity was slightly different from the (simplified) one given here; it was not based on the bounded functions but on the projection valued measure $E_{\triangle}$, where $\triangle$ is a Borel subset of the configuration space, and a system of imprimitivity referred to the pair $\left(U(g), E_{\triangle}\right)$. In terms of these the imprimitivity relation (2.8) takes the form $U(g) E_{\triangle} U^{-1}(g)=E_{g} \triangle$, where $g \triangle$ is the translated Borel set. For the systems we consider in this paper, this description in terms of projection valued measures can be seen to be equivalent to the one based on the bounded functions. Indeed the projection valued measure $E_{\triangle}$ can be recovered from (2.7) since $E_{\triangle} \equiv \int d^{3} q \chi_{\triangle}(q)|q\rangle\langle q|$, where $\chi_{\triangle}$ is the characteristic functions for the Borel subset $\triangle$ of the configuration space.

3 In order to continue using bounded functions in place of projection valued measure in the imprimitivity relation we need to assume that the action of $\mathrm{G}$ on the coset space $\mathrm{G} / \mathrm{H}$ is continuous and that $\mathrm{G} / \mathrm{H}$ is locally compact Hausdorff and satisfies the second axiom of countability (see lemma 9.1 in Ref. 14). This will be the case if G compact, or locally compact and abelian, or a (semidirect) product of such groups. 
to a system - reflecting the fact that the identification of a coset space as $\mathrm{G} / \mathrm{H}$ is far from unique. Thus the choice of a specific system of imprimitivity must be guided by additional requirements such as symmetry or dynamics.

Now that the imprimitivity relation (2.8) is taken to be the basis upon which the quantum theory of a system with configuration space $\mathrm{G} / \mathrm{H}$ is constructed, we would like to know as much as we can about the irreducible, unitary representations of this relation. In what follows we shall show that, in contrast to the Stone--von Neumann theorem, there are now many inequivalent representations of (2.8) possible, labelled by the irreducible unitary representations of $\mathrm{H}$, and hence many inequivalent quantizations for a system based on $\mathrm{G} / \mathrm{H}$. In order to do this we first need to recall some properties of coset spaces.

To fix the geometry of the coset space (and our notation), the group $\mathrm{G}$ is taken to act on the left, hence the coset space $\mathrm{G} / \mathrm{H}$ is identified with the left cosets $\{g \mathrm{H} \mid g \in \mathrm{G}\}$. One can regard $\mathrm{G}$ as the principal fibre bundle $\mathrm{G}(\mathrm{G} / \mathrm{H}, \mathrm{H})$ with the base space $\mathrm{G} / \mathrm{H}$, the fibre $\mathrm{H}$ and the projection from $\mathrm{G}$ to $\mathrm{G} / \mathrm{H}$ being $\operatorname{pr}(g):=g \mathrm{H}$. Note that for the left action of G we have $\operatorname{pr}\left(g_{1} g_{2}\right)=g_{1} \operatorname{pr}\left(g_{2}\right)$, while for the right action of $\mathrm{H}$ we have $\operatorname{pr}(g h)=\operatorname{pr}(g)$. A section $\sigma(q)$ is a map $\sigma: \mathrm{G} / \mathrm{H} \mapsto \mathrm{G}$ such that $\operatorname{pr}(\sigma(q))=q$. We just require that the map $\sigma$ be measurable, allowing $\sigma$ to be defined only locally as a continuous section. Given a section $\sigma$ then $g \sigma(q)$ is an element in the fibre above $g q$, and hence we have $h_{\sigma}(g, q) \in \mathrm{H}$ such that

$$
g \sigma(q)=\sigma(g q) h_{\sigma}(g, q) .
$$

If $\sigma^{\prime}$ is another section then $\sigma^{\prime}(q)=\sigma(q) \tilde{h}(q)$ for some $\tilde{h}(q) \in$ H. It then follows that under a change of section we have

$$
h_{\sigma^{\prime}}(g, q)=\tilde{h}^{-1}(g q) h_{\sigma}(g, q) \tilde{h}(q) .
$$

When it is clear, or not too important, which section is involved we shall simply write $h_{\sigma}(g, q)$ as $h(g, q)$. As well as this behaviour (2.10) under the change of section, $h(g, q)$ also satisfies the condition $h(1, q)=1$ and the cocycle condition

$$
h\left(g_{1} g_{2}, q\right)=h\left(g_{1}, g_{2} q\right) h\left(g_{2}, q\right) .
$$

The coset space $\mathrm{G} / \mathrm{H}$ has a naturally defined G-invariant metric induced from the Killing metric on G (see Appendix C for full definitions). Thus there is a G-invariant measure $D q$ on $\mathrm{G} / \mathrm{H}$ which can be characterized by

$$
\int_{\mathrm{G}} D g F(g)=\int_{\mathrm{G} / \mathrm{H}} D q \int_{\mathrm{H}} D h F(\sigma(q) h),
$$


where $F$ is an arbitrary function on $\mathrm{G}$ and $D g(D h)$ is the Haar measure on $\mathrm{G}(\mathrm{H})$.

Given an irreducible unitary representation $\chi$ of $\mathrm{H}$ on a Hilbert space $\mathcal{H}_{\chi}$, we can construct the Hilbert space $\mathcal{H}$ over $\mathrm{G} / \mathrm{H}$ consisting of $\mathcal{H}_{\chi}$-valued state vectors on $\mathrm{G} / \mathrm{H}$, i.e.,

$$
\mathcal{H} \simeq L^{2}\left(\mathrm{G} / \mathrm{H}, \mathcal{H}_{\chi}\right)
$$

Given such a ket $|\psi\rangle \in \mathcal{H}$, we can find its components in the standard way; we use the position basis $|q\rangle$ for $\mathrm{G} / \mathrm{H}$ and the finite dimensional orthonormal basis $|\chi, \mu\rangle$ for $\mathcal{H}_{\chi}$ (for notations, see Appendix A) and define the vector-valued wave functions to be

$$
\psi_{\mu}(q)=(\langle q|\otimes\langle\chi, \mu|) \mid \psi\rangle=\langle q, \chi, \mu \mid \psi\rangle
$$

The inner-product on these states is constructed from the pairing on $\mathcal{H}_{\chi}$ and the Ginvariant measure $D q$ on $\mathrm{G} / \mathrm{H}$ :

$$
\langle\phi \mid \psi\rangle=\int_{\mathrm{G} / \mathrm{H}} D q\langle\phi(q) \mid \psi(q)\rangle_{\mathcal{H}_{\chi}} .
$$

On these vector-valued wave functions we can define the unitary induced representation $U(g)$ of (the left action of) G by

$$
(U(g) \psi)_{\mu}(q)=\sum_{\nu} \pi_{\mu \nu}^{\chi}\left(h_{\sigma}\left(g, g^{-1} q\right)\right) \psi_{\nu}\left(g^{-1} q\right)
$$

where the matrix elements of the unitary operator $\pi^{\chi}(h)$, implementing the irreducible representation $\chi$, are

$$
\pi_{\mu \nu}^{\chi}(h):=\left\langle\chi, \mu\left|\pi^{\chi}(h)\right| \chi, \nu\right\rangle,
$$

and a choice of section has been made. The fact that this is a representation follows from the cocycle condition (2.11).

In the representation $U(g)$ in (2.16) we have suppressed reference to the choice of section $\sigma$ since a different choice of section leads to a unitarily equivalent representation. To see this we write $U(g)=U_{\sigma}(g)$, to expose the $\sigma$-dependence explicitly, and consider the unitary mapping $T: \mathcal{H} \rightarrow \mathcal{H}$ given by

$$
(T \psi)_{\mu}(q)=\sum_{\nu} \pi_{\mu \nu}^{\chi}(\tilde{h}(q)) \psi_{\nu}(q)
$$


where $\tilde{h}(q)$ is the element of $\mathrm{H}$ relating the section $\sigma$ to the section $\sigma^{\prime}$ as in (2.10). Then one can readily confirm that $T$ intertwines the two representations,

$$
\left(U_{\sigma}(g) T \psi\right)_{\mu}(q)=\left(T U_{\sigma^{\prime}}(g) \psi\right)_{\mu}(q)
$$

hence they are unitarily equivalent.

It is now clear that this unitary representation of $\mathrm{G}$ - induced from the irreducible representation $\chi$ of $\mathrm{H}$ - provides us with a representation of the imprimitivity relation (2.8) since, for any bounded function $F(q)$ on $\mathrm{G} / \mathrm{H}$,

$$
\begin{aligned}
\left(U(g) \widehat{F}(q) U^{-1}(g) \psi\right)_{\mu}(q) & =\sum_{\nu} \pi_{\mu \nu}^{\chi}\left(h\left(g, g^{-1} q\right)\right)\left(\widehat{F}(q) U^{-1}(g) \psi\right)_{\nu}\left(g^{-1} q\right) \\
& =\sum_{\nu} \pi_{\mu \nu}^{\chi}\left(h\left(g, g^{-1} q\right)\right) F\left(g^{-1} q\right)\left(U^{-1}(g) \psi\right)_{\nu}\left(g^{-1} q\right) \\
& =\sum_{\nu, \lambda} \pi_{\mu \nu}^{\chi}\left(h\left(g, g^{-1} q\right)\right) F\left(g^{-1} q\right) \pi_{\nu \lambda}^{\chi}\left(h\left(g^{-1}, q\right)\right) \psi_{\lambda}(q) \\
& =\sum_{\lambda} \pi_{\mu \lambda}^{\chi}\left(h\left(g, g^{-1} q\right) h\left(g^{-1}, q\right)\right) F\left(g^{-1} q\right) \psi_{\lambda}(q) \\
& =F\left(g^{-1} q\right) \psi_{\mu}(q) \\
& =\left(\widehat{F}\left(g^{-1} q\right) \psi\right)_{\mu}(q) .
\end{aligned}
$$

The imprimitivity theorem due to Mackey then states that these induced representations provide all the distinct irreducible representations of the imprimitivity relation (2.8). Thus we see that, in contrast to the uniqueness of the Schrödinger representation based on the Weyl relations (2.2), (2.3), there are many inequivalent quantizations possible when the configuration space is identified with the coset space $\mathrm{G} / \mathrm{H}$, and that these inequivalent quantizations are labelled by the irreducible unitary representations of the group $\mathrm{H}$.

Returning to the example of $\mathbb{R}^{3}$, which we identified with the coset space $\mathrm{E}(3) / \mathrm{SO}(3)$, we see that there are now inequivalent quantizations labelled by the irreducible unitary representations of $\mathrm{SO}(3)$. Alternatively, to avoid the need to consider projective representations of (2.8), we may work with the universal covering group $\widetilde{\mathrm{E}(3)} \equiv \mathrm{SU}(2) \ltimes \mathbb{R}^{3}$, thus identifying $\mathbb{R}^{3}$ with the coset $\widetilde{\mathrm{E}(3)} / \mathrm{SU}(2)$. Hence it is the positive (half-) integers labelling the irreducible unitary representations of $\mathrm{SU}(2)$ that characterize the possible quantizations. Reverting to more familiar notation, we know that, for each half-integer $j$, there is an irreducible representation $\pi^{j}=D^{j}$ of $\mathrm{SU}(2)$ on the Hilbert space $\mathcal{H}_{j} \simeq \mathbb{C}^{2 j+1}$. Thus 
the quantization of this system of imprimitivity corresponding to the irreducible representation of $\mathrm{SU}(2)$ labelled by $j$ is defined on the Hilbert space $\mathcal{H} \simeq L^{2}\left(\mathbb{R}^{3}, \mathbb{C}^{2 j+1}\right)$. Denoting $g \in \widetilde{\mathrm{E}(3)}$ by $g=(h, a)$ where $h \in \mathrm{SU}(2), a \in \mathbb{R}^{3}$, and choosing the section $\sigma(q)=(1, q)$, we see from (2.16) that the representation of $\widetilde{\mathrm{E}(3)}$ in this quantization is given by

$$
(U(h, a) \psi)_{m}(q)=\sum_{n=-j}^{j} D_{m n}^{j}(h) \psi_{n}\left(h^{-1}(q-a)\right) .
$$

The Schrödinger representation (2.4) simply corresponds to the case $j=0$, that is, the trivial representation of $\mathrm{SU}(2)$.

The unitary representation (2.16) of $\mathrm{G}$ that arises in the system of imprimitivity allows us to define left-momentum operators $\widehat{L}_{m}(m=1, \ldots, \operatorname{dim} \mathrm{G})$ by

$$
\left(\widehat{L}_{m} \psi\right)_{\mu}(q):=\frac{d}{d t}\left(U\left(e^{t T_{m}}\right) \psi\right)_{\mu}(q)_{\left.\right|_{t=0}}
$$

where $\left\{T_{m}\right\}$ is a basis of the Lie algebra $\mathfrak{g}$ of $\mathrm{G}$ and $e^{t T_{m}}$ is the exponential mapping on G. This representation of the Lie algebra $\mathfrak{g}$ can be extended to the enveloping algebra, allowing us to construct observables polynomial in the momentum. We note that when this identification of momentum is applied to the representation $(2.21)$ of $\widetilde{\mathrm{E}(3)}$ we recover, in addition to the observables corresponding to the generators of translations $\hat{p}_{\alpha}$, new basic

observables $\widehat{S}_{i}(i=1,2,3)$ satisfying $\left[\widehat{S}_{i}, \widehat{S}_{j}\right]=i \varepsilon_{i j k} \widehat{S}_{k}$. Clearly, $\widehat{S}_{i}$ can be regarded as spin in the quantum theory; in fact, this account of spin based on inequivalent quantizations was one of the prime motives for Mackey's original analysis [6].

\subsection{Dynamics and the H-connection}

Having addressed the kinematical aspects associated with the quantization of a system whose configuration space $Q$ is $\mathrm{G} / \mathrm{H}$, we now wish to discuss how the dynamics is described in the various inequivalent quantizations. For simplicity we shall consider the Hamiltonian leading to a free particle moving on the coset $\mathrm{G} / \mathrm{H}$. But before discussing a generic coset $\mathrm{G} / \mathrm{H}$, let us recall that in the $\mathrm{G} / \mathrm{H}=\widetilde{\mathrm{E}(3)} / \mathrm{SU}(2) \simeq \mathbb{R}^{3}$ case the Hamiltonian for a free particle is identified with the operator $\widehat{H}=\frac{1}{2} \hat{p}_{\alpha} \hat{p}_{\alpha}$, and this can be interpreted as the representation furnished by (2.22) for the quadratic Casimir operator for $\widetilde{\mathrm{E}(3)}$ restricted to its abelian, normal subgroup $\mathbb{R}^{3}$. Similarly, for a generic $G / H$ with compact $G$, the Hamiltonian $\widehat{H}$ may be identified with the quadratic Casimir of the group G; indeed, it 
is known that the classical Hamiltonian $H$, that corresponds to that $\widehat{H}$, generates free (geodesic) motion on $\mathrm{G} / \mathrm{H}$. Thus, for such systems, the Hamiltonian is given by

$$
\widehat{H}=\frac{1}{2} \eta^{m n} \widehat{L}_{m} \widehat{L}_{n}
$$

where $\eta^{m n}$ is the inverse of $\eta_{m n}:=\operatorname{Tr}\left(T_{m} T_{n}\right)$. In passing we note that considering a Hamiltonian for non-free particle by adding potential energy terms to (2.23) does not siginificantly alter the conclusions we shall reach.

Through an analysis of the form of the Hamiltonian (2.23), Landsman and Linden [10] (see also $[15,16,17,18]$ ) made the important observation that there is a 'fictitious' connection that couples to the particle in the non-trivial quantum sectors among the inequivalent quantizations. More precisely, they showed that in the Hamiltonian (2.23) one can isolate the quadratic Casimir for the group $\mathrm{H}$ which becomes constant on a irreducible representation $\chi$. Then, using some local set of coordinates on $\mathrm{G} / \mathrm{H}$, the Hamiltonian can be written (up to the constant) in the gauge covariant form,

$$
\widehat{H}=-\frac{1}{2} g^{\alpha \beta}\left(\nabla_{\alpha}+A_{\alpha}\right)\left(\partial_{\beta}+A_{\beta}\right),
$$

where $\alpha=1, \ldots, \operatorname{dim}(\mathrm{G} / \mathrm{H}), \nabla_{\alpha}$ is the covariant derivative construced out of the natural G-invariant metric $g_{\alpha \beta}$ on $\mathrm{G} / \mathrm{H}$, and the connection $A_{\alpha}$ is the canonical $\mathrm{H}$-connection. Note that in a non-trivial sector (corresponding to a non-trivial representation $\chi$ ) the Hamiltonian $\widehat{H}$ is a matrix-valued $\left(\pi^{\chi}(\mathfrak{h})\right.$-valued) vector field on $\mathrm{G} / \mathrm{H}$, while in the trivial sector it reduces to the Laplace-Beltrami operator, $\widehat{H}=-\frac{1}{2} \triangle_{\mathrm{LB}}=-\frac{1}{2} g^{\alpha \beta} \nabla_{\alpha} \partial_{\beta}$, as required. The precise definition, and properties, of the H-connection will be given in detail later, but for now let us mention a simple example where the connection becomes a familiar object. Consider a particle moving freely on the two-sphere $S^{2}$. The configuration space can be identified with the coset space $S^{2} \simeq \mathrm{SO}(3) / \mathrm{SO}(2)$, thus the quantum theories will be characterized by an integer $n$ labelling the irreducible unitary representations of $\mathrm{SO}(2)$. The resulting $\mathrm{SO}(2)$ connection on $S^{2}$ can be identified with that of a Dirac monopole centred at the origin of the two-sphere. Thus, for this specific example, we can interpret the different possible quantum theories as those that describe the particle coupled to a Dirac monopole of magnetic charge $n$ (for more details, see Sect. 6.3). 


\subsection{Other approaches and problems}

Mackey's account of quantization is, albeit the first, just one of several approaches to the quantization of a classical system whose configuration space is more complicated than $\mathbb{R}^{n}$. As we mentioned in the Introduction, these other approaches include geometric quantization [7], the canonical group approach [8] and a $\mathrm{C}^{*}$-algebra approach $[9,10]$. These have their own virtues but for the coset spaces $G / H$ they all reproduce the inequivalent quantizations described by Mackey through his system of imprimitivity. Take for example a particle moving freely on the two-sphere $S^{2}$. The methods of geometric quantization, which gives an account of the transition from a classical to a quantum system based on the (symplectic) geometry of the phase space, predicts many quantizations labelled by the cohomology group $H^{2}\left(S^{2}, \mathbb{Z}\right) \simeq \mathbb{Z}$ classifying the possible prequantum line bundles on the phase space $T^{*} S^{2}$ (the cotangent bundle of $S^{2}$ ). If we follow the canonical group approach [8] and exploit the fact that the phase space $T^{*} S^{2}$ is, in fact, a homogeneous symplectic manifold upon which the (canonical) group E(3) acts transitively and symplectically, then, the possible quantizations will correspond to the irreducible representations of the canonical group E(3) which, by Mackey's theory of induced representations applied to such a semi-direct product group [6], are labelled by the irreducible representations of the isotropy (little) group $\mathrm{SO}(2)$. The connection between geometric quantization, the canonical group approach and Mackey's approach becomes even clearer when we recall that the irreducible representations of $\mathrm{E}(3)$ are geometrically characterized by the coadjoint orbits of $\mathrm{E}(3)$. Generically, these orbits are precisely the manifold $T^{*} S^{2}$, but with a non-standard symplectic form corresponding to that describing the minimal coupling of a particle with a Dirac monopole (see page 14 in [19]). So we see that, at least for the simple model of a particle on $S^{2}$, there seems to be a consensus in the literature that many inequivalent quantizations are possible, and that these different quantum sectors come equipped with a topological abelian gauge field. For the more general coset space we can again argue that Mackey's account of the inequivalent quantizations will at least be contained in the other approaches since the phase space $T^{*}(\mathrm{G} / \mathrm{H})$ are also homogeneous symplectic manifolds [8]. It is, however, important to note that the representation theory of the appropriate canonical group, or, equivalently, geometric quantization applied to the various coadjoint orbits, will not always result in the form of vector-valued wave functions on the original coset space, thus we must always keep it in mind that it is possible for more to happen in the process of quantizing a classical system than Mackey would suggest. (For more details on this point we refer to $[8,10]$.) 
The approaches to quantization discussed above all take as basic the need to generalize the canonical commutation relations (2.1), and thus can be characterized as being, at heart, 'operator descriptions'. As such they lack the physical appeal of the path-integral approach to quantization and, hence, suffer from all the problems inherent in such operator formalisms when one tries to generalize these quantum mechanical results to field theory. In particular, the vector-valued nature of the wave functions forces the directly developed path-integral [10] to be unattractive; it is a type of path-ordered, discrete path-integral which leads to a transition matrix rather than an amplitude. In fact, such a problem was encountered earlier when spin was incorporated into the path-integral [20], where, in order to avoid the use of the discrete path-integral, an attempt was made to develop a continuous path-integral for spin. Thus it will be natural to illustrate the idea of our reformulation of Mackey's approach by showing how spin can emerge in the context of quantizing on a coset, and how the continuous path-integral can be developed which reproduces all the results that the operator descriptions provide. This will be the subject of the next section. 


\section{Spin without vector-valued wave functions}

In this section an account of spin will be presented that views the coset space $\mathbb{R}^{3} \simeq$ $\widetilde{\mathrm{E}(3)} / \mathrm{SU}(2)$ as a constrained system on $\widetilde{\mathrm{E}(3)}$. This will be seen to be equivalent to Mackey's description in terms of a system of imprimitivity, but avoids the use of vector-valued wave functions. The path-integral version of the construction will be seen to recover the continuous spin description of Nielsen and Rohrlich [13], although the argument for the quantization of spin will be different. The methods developed in this section will form the basis for our generalization of Dirac's approach to include the possibility of inequivalent quantizations.

\subsection{An operator description of spin}

We have seen that through Mackey's analysis, spin enters into the quantum theory once the configuration space $Q=\mathbb{R}^{3}$ is identified with the coset space $\widetilde{\mathrm{E}(3)} / \mathrm{SU}(2)$. The resulting quantum states become vector-valued wave functions, and new basic observables $\widehat{S}_{i}$ emerge which are identified with the spin. In this account spin arises only in the quantum theory - no classical counterpart exists. However, from a path-integral point of view, it is desirable to avoid using vector-valued wave functions and, instead, introduce an (effective) classical system whose quantization leads to the observables $\widehat{S}_{i}$ and the finite dimensional Hilbert spaces $\mathcal{H}_{j} \simeq \mathbb{C}^{2 j+1}$.

The first attempt to develop such a classical account of spin was by Schulman [20]. Focusing purely on the spin degrees of freedom, we see that the group $\mathrm{SU}(2)$ can itself be taken to be a configuration space of a system that has momentum variables obeying the $\mathrm{su}(2)$ algebra, and thus a good candidate for spin. Applying Mackey's quantization procedure to this system (where now $\mathrm{G} / \mathrm{H}=\mathrm{SU}(2) /\{1\}$ ) we get a single quantization in which the state space is identified with the wave functions on $\mathrm{SU}(2): \mathcal{H} \simeq L^{2}(\mathrm{SU}(2))$. The unitary representation $(2.16)$ of $\mathrm{SU}(2)$ is now the left-regular representation

$$
\left(U_{\mathrm{L}}(\tilde{g}) \psi\right)(g)=\psi\left(\tilde{g}^{-1} g\right)
$$

where both $\tilde{g}$ and $g$ are elements of SU(2). The generators $\widehat{L}_{i}(i=1,2,3)$ of this left action satisfy the $\mathrm{su}(2)$ commutator relations

$$
\left[\widehat{L}_{i}, \widehat{L}_{j}\right]=i \varepsilon_{i j k} \widehat{L}_{k}
$$


Although this quantum theory proposed by Schulman has basic observables satisfying the commutator relations for spin, it is not a satisfactory account since the state space is not finite dimensional. In fact all irreducible representations of $\mathrm{SU}(2)$ (and hence all possible total spins, not just a specific one) are contained in the Hilbert space $L^{2}(S U(2))$. This result follows at once from the Peter-Weyl theorem applied to SU(2), which we now summarize.

The content of the Peter-Weyl theorem [21] is that the matrix elements $D_{m n}^{j}(g):=$ $\left\langle j, m\left|D^{j}(g)\right| j, n\right\rangle$, of the irreducible representations $D^{j}(g)$ of $\mathrm{SU}(2)$, form an orthonormal basis for the wave functions on $\mathrm{SU}(2)$. Thus any wave function $\psi(g) \in L^{2}(\mathrm{SU}(2))$ can be written as

$$
\psi(g)=\sum_{j} \sum_{m=-j}^{j} \sum_{m^{\prime}=-j}^{j} C_{m m^{\prime}}^{j} D_{m m^{\prime}}^{j *}(g),
$$

where the matrix elements satisfy the orthonormal relations

$$
\int_{\mathrm{SU}(2)} D g D_{m m^{\prime}}^{j *}(g) D_{n n^{\prime}}^{j}(g)=\frac{1}{2 j+1} \delta_{m n} \delta_{m^{\prime} n^{\prime}}
$$

and $D g:=\prod_{i=1}^{3}\left(g^{-1} d g\right)^{i}$ is the Haar measure on $\mathrm{SU}(2)$. Thus we see that the wave functions $\psi(g)$ do contain all spins $j$ in general. Incidentally, we note that the use of $D_{m m^{\prime}}^{j *}(g)$ in (3.3) is forced on us by the requirement that we wish to construct wave functions which transform correctly under the left action of $\mathrm{SU}(2)$, namely, we wish to have the identities

$$
\begin{aligned}
\widehat{L}_{3} D_{m m^{\prime}}^{j *}(g) & =m D_{m m^{\prime}}^{j *}(g) \\
\widehat{L}_{ \pm} D_{m m^{\prime}}^{j *}(g) & =\sqrt{(j \mp m)(j \pm m+1)} D_{m \pm 1, m^{\prime}}^{j *}(g)
\end{aligned}
$$

To improve upon Schulman's analysis we must find some means of isolating a finite dimensional subspace of these wave functions which carries a single irreducible representation of $\mathrm{SU}(2)$. To facilitate in this we recall that, as well as the left action of $\mathrm{SU}(2)$ on itself, there is also a right action which leads to the right-regular representation of $\mathrm{SU}(2)$,

$$
\left(U_{\mathrm{R}}(\tilde{g}) \psi\right)(g):=\psi(g \tilde{g})
$$

The generators of this right action, which we denote by $\widehat{R}_{i}$, also satisfy the $\mathrm{su}(2)$ commutator relations

$$
\left[\widehat{R}_{i}, \widehat{R}_{j}\right]=i \varepsilon_{i j k} \widehat{R}_{k}
$$


and commute with the generators of the left action

$$
\left[\widehat{R}_{i}, \widehat{L}_{j}\right]=0
$$

Analogously to (3.5), acting on the wave functions $D_{m n}^{j *}(g)$ we have the identities

$$
\begin{aligned}
& \widehat{R}_{3} D_{m m^{\prime}}^{j *}(g)=m^{\prime} D_{m m^{\prime}}^{j *}(g) \\
& \widehat{R}_{ \pm} D_{m m^{\prime}}^{j *}(g)=\sqrt{\left(j \pm m^{\prime}\right)\left(j \mp m^{\prime}+1\right)} D_{m, m^{\prime} \mp 1}^{j *}(g)
\end{aligned}
$$

From (3.3) and (3.9) we see that the wave functions on $\mathrm{SU}(2)$ which satisfy the two conditions

$$
\widehat{R}_{3} \psi(g)=j \psi(g) \quad \text { and } \quad \widehat{R}_{-} \psi(g)=0,
$$

are of the form

$$
\psi(g)=\sum_{m=-j}^{j} C_{m j}^{j} D_{m j}^{j *}(g) .
$$

Thus the solutions to (3.10) span a finite dimensional Hilbert space (of dimension $(2 j+1)$ ) with basis vectors given by the wave functions $\left\{D_{m j}^{j *}(g)\right\}(m=-j, \ldots, j)$. Due to (3.8), the operators $\widehat{L}_{i}$ preserve these states and hence, on this subspace, provide an irreducible representation of $\mathrm{SU}(2)$ with highest weight (total spin) $j$. The generators of spin, $\widehat{S}_{i}$, are thus identified with the restriction to these states of the generators $\widehat{L}_{i}$ of the left-regular representation of $\mathrm{SU}(2)$.

The conclusion of this analysis is that the quantum description of spin emerges from the system of $\mathrm{SU}(2)$ subject to the restrictions (3.10) on the states. This operator description of spin uses wave functions on $\mathrm{SU}(2)$ subject to the conditions (3.10), hence a traditional path-integral formulation should be possible.

\subsection{A path-integral description of spin}

To describe the dynamics on $\mathrm{SU}(2)$, let us work in a specific matrix representation, the

defining representation of $\mathrm{SU}(2)$, and take for a basis $T_{i}:=\frac{\sigma_{i}}{2 i}$ for which $\operatorname{tr}\left(T_{i} T_{j}\right)=-\frac{1}{2} \delta_{i j}$ and $\left[T_{i}, T_{j}\right]=\epsilon_{i j k} T_{k}$. Then, for a free particle on $\mathrm{SU}(2)$, the transition amplitude from an initial point $g_{0}$ at time $t=0$ to a final point $g_{1}$ at time $t=T$ is represented by the path-integral

$$
Z=\int \mathcal{D} g \exp \left(-i \int_{0}^{T} d t \operatorname{tr}\left(g^{-1} \dot{g}\right)^{2}\right)
$$


where $\mathcal{D} g:=\prod_{t} D g(t)$, and the formal sum is over all possible paths $g(t)$ such that $g(0)=g_{0}$ and $g(T)=g_{1}$. Conditions (3.10) suggest that, in order to recover the pathintegral description of spin, we must impose constraints associated with the right action, and for this purpose we consider the phase space version of (3.12),

$$
Z=\int \mathcal{D} g \mathcal{D} R \exp \left(i \int_{0}^{T} d t\left(2 \operatorname{tr} R\left(g^{-1} \dot{g}\right)+\operatorname{tr} R^{2}\right)\right)
$$

The measure for the momentum variables is taken to be $\mathcal{D} R=\prod_{t} \prod_{i=1}^{3} d R_{i}(t)$ where the components $R_{i}:=-2 \operatorname{tr}\left(R T_{i}\right)$ are the classical right momenta which generate the right action on $\mathrm{SU}(2)$ (more details on this identification will be presented in Sect. 4 and 5, for now all we really need to note is that by integrating out the momentum in (3.13) one recovers $(3.12))$.

We now need to determine the effective classical counterpart to the conditions (3.10) to be used in the path-integral description of spin. We cannot simply deduce that $R_{3}=j$ and $R_{+}=0$, since $R_{+}$is not an element of $\mathrm{su}(2)$. However, taking expectation values in the states $(3.11)$ we can deduce that $\left\langle\widehat{R}_{3}\right\rangle=j$, while $\left\langle\widehat{R}_{1}\right\rangle=\left\langle\widehat{R}_{2}\right\rangle=0$. This suggests that we should take the effective classical version of (3.10) to be

$$
R_{3}=j \quad \text { and } \quad R_{1}=R_{2}=0
$$

The Poisson bracket relations among the right momenta are $\left\{R_{i}, R_{j}\right\}=\epsilon_{i j k} R_{k}$ (for this derivation, see Appendix B), hence, from (3.14), we see that (for $j \neq 0$ ) these represent a mixed set of constraints with $R_{3}-j$ being first class while $R_{1}$ and $R_{2}$ are second class. From this it is clear that the classical set of constraints (3.14) leads to the quantum conditions (3.10) since these represent the 'maximal first class subalgebra' constructed out of the constraints (3.14) (modulo an ambiguity in choosing the set). If $j=0$ then all the constraints are first class and we reduce to a point, which is consistent with the quantum theory where the states are just the constant functions on $\mathrm{SU}(2)$.

Restricting to the situation when $j \neq 0$, we note that the first class constraint function $R_{3}-j$ will generate a $\mathrm{U}(1)$-action

$$
g \longrightarrow g s, \quad \text { where } \quad s=e^{\theta T_{3}}
$$

as a gauge symmetry. Combining with the second class constraints, we see that the original phase space $T^{*}(\mathrm{SU}(2))=\mathrm{SU}(2) \times \mathbb{R}^{3}$ is now reduced to the compact phase space 
$\mathrm{SU}(2) / \mathrm{U}(1) \simeq S^{2}$. It is this compactness of the phase space that results in the finite dimensional Hilbert space; indeed, the phase space $S^{2}$ was postulated from the outset by Nielsen and Rohrlich in their path-integral description of spin.

A path-integral account of the system (3.13), subject to the constraints (3.14), is now quite straightforward to develop. The constraints (3.14) imply that the measure in (3.13) is modified [22] resulting in the constrained path-integral

$$
\begin{aligned}
Z_{\text {spin }}=\int \mathcal{D} g \mathcal{D} R \delta\left(R_{1}\right) \delta\left(R_{2}\right) & \delta\left(R_{3}-j\right) \delta(\xi)\left|\left\{R_{3}, \xi\right\}\right| \\
& \times \exp \left(i \int_{0}^{T} d t\left(2 \operatorname{tr} R\left(g^{-1} \dot{g}\right)+\operatorname{tr} R^{2}\right)\right),
\end{aligned}
$$

where $\xi=0$ is some gauge fixing condition for the constraint $R_{3}=j$ which, for simplicity, is taken to be a function of the configuration space variables only so that the factor $\left|\left\{R_{3}, \xi\right\}\right|$ be independent of $R_{i}$. Performing the momentum integrals then yields

$$
Z_{\text {spin }}=\int \mathcal{D} g \delta(\xi)\left|\left\{R_{3}, \xi\right\}\right| \exp \left(i \int_{0}^{T} d t 2 j \operatorname{tr} T_{3}\left(g^{-1} \dot{g}\right)\right) .
$$

Changes in the gauge fixing are implemented by the U(1)-action (3.15). When applying the gauge transformation $g(t) \rightarrow g(t) s(t)$ within the path-integral we must preserve the boundary conditions on the paths. This implies $s(T)=s(0)=1$ or, in view of the basis we are using,

$$
\theta(T)-\theta(0)=4 \pi n
$$

for some integer $n$. On the other hand, under the transformation (3.15) the Lagrangian in the path-integral (3.17),

$$
L_{\text {spin }}=2 j \operatorname{tr} T_{3}\left(g^{-1} \dot{g}\right) \text {, }
$$

changes to

$$
L_{\text {spin }} \longrightarrow L_{\text {spin }}-j \dot{\theta} \text {. }
$$

Thus, in order for the path-integral (3.17) to be independent of the gauge fixing condition $\xi$ (within the class of such gauge fixing connected by the transformation $(3.15))^{4}$ we must ensure that $e^{-i \int d t j \dot{\theta}}=1$, or

$$
j(\theta(T)-\theta(0))=2 \pi \times \text { integer } .
$$

\footnotetext{
4 There is a subtlety with the gauge independence of the path-integral due to the
} existence of a Gribov ambiguity; however, we shall not address this point here (see [23]). 
For this to be true for any $n$ in (3.18) we must have $2 j \in \mathbb{Z}$, and hence we have recovered the quantization condition for spin. (Recall that there is a two to one correspondence between $\mathrm{SU}(2)$ and $\mathrm{SO}(3)$, in particular $1_{\mathrm{SO}(3)}=\{1,-1\}_{\mathrm{SU}(2)}$. Hence, had we been working on $\mathrm{SO}(3)$, the condition (3.18) would be replaced by $\theta(T)-\theta(0)=2 \pi n$, and we would recover the integer quantization for spin $j \in \mathbb{Z}$ in that case.)

A useful local set of coordinates on $\mathrm{SU}(2)$ are the Euler angles $(\alpha, \beta, \gamma)$, where $g=$ $e^{\alpha T_{3}} e^{\beta T_{2}} e^{\gamma T_{3}}(0 \leq \alpha<2 \pi, 0 \leq \beta \leq \pi, 0 \leq \gamma<4 \pi)$. In terms of these the path-integral (3.17) becomes

$$
Z_{\text {spin }}=\int \mathcal{D} \alpha \mathcal{D} \beta \mathcal{D} \gamma \sin \beta \delta(\xi)\left|\frac{\partial \xi}{\partial \gamma}\right| \exp \left(-i \int_{0}^{T} d t j(\dot{\alpha} \cos \beta+\dot{\gamma})\right)
$$

A gauge fixing that is only ill defined at the south pole $(\beta=\pi)$ is given by

$$
\xi_{+}= \begin{cases}\gamma+\alpha, & \text { if } 0 \leq \gamma<2 \pi \\ \gamma-2 \pi+\alpha, & \text { if } 2 \pi \leq \gamma<4 \pi\end{cases}
$$

This leads to the path-integral

$$
Z_{\text {spin }}=\int \mathcal{D} \alpha \mathcal{D} \beta \sin \beta \exp \left(i \int_{0}^{T} d t j \dot{\alpha}(1-\cos \beta)\right) .
$$

The Lagrangian $j \dot{\alpha}(1-\cos \beta)$ is well defined (actually zero) at the north pole, while at the south pole it becomes $2 j \dot{\alpha}$. Paths going $n$-times around this singular point contribute $2 j \times 2 \pi n$ to the action, which is an irrelevant phase since $2 j$ is an integer. This result is also expected since one can use the gauge invariance (3.15) to go from the gauge fixing condition (3.23) to

$$
\xi_{-}= \begin{cases}\gamma-\alpha, & \text { if } 0 \leq \gamma<2 \pi \\ \gamma-2 \pi-\alpha, & \text { if } 2 \pi \leq \gamma<4 \pi\end{cases}
$$

which is only ill defined at the north pole.

Expression (3.24) is equivalent to that proposed by Nielsen and Rohrlich [13] (see also [24]) for a path-integral description of spin. Here we have seen that it can be derived from a constrained path-integral formulation on $\mathrm{SU}(2)$. The point to be observed is that the above construction admits an interpretation in terms of the generalized Dirac approach. Indeed, the conditions (3.10) on which our constrained path-integral is based are of the type (1.4) and hence, following the generalized Dirac approach, they represent the allowed anomalous behaviour for this system starting with the (classical) first class constraints given by (3.14) with $j=0$, which are of the type (1.1). Furthermore, the quantization 
of spin $j$ is correctly deduced from the consistency of the path-integral. In other words, using the generalized Dirac approach one can construct the quantum mechanics of spin by quantizing on the trivial coset $\mathrm{G} / \mathrm{H}=\mathrm{SU}(2) / \mathrm{SU}(2)$. It is now evident that, reinstating the abelian group $\mathbb{R}^{3}$ to turn $\mathrm{SU}(2)$ into $\widetilde{\mathrm{E}}(3)$, we can obtain spin (in addition to the $\mathbb{R}^{3}$ degrees of freedom) by quantizing on $Q=\mathbb{R}^{3}$ identified with the coset $\widetilde{\mathrm{E}(3)} / \mathrm{SU}(2)$, which is the result Mackey obtained in his approach using the system of imprimitivity based on $\widetilde{\mathrm{E}(3)} / \mathrm{SU}(2)$. In this case, the corresponding path-integral describing a particle moving in $\mathbb{R}^{3}$ with spin has an effective phase space $T^{*} \mathbb{R}^{3} \times S^{2}$, where the two-sphere has radius $j^{2}$.

We have seen that the classical spinless particle, described by the first class constraints (3.14) with $j=0$, can acquire spin upon quantization by the change in the structure of the constraints, which is required if we are to recover Mackey's result. Such a change in the constraints is usually anathema to the whole constrained formalism; indeed the avoidence of this is one of the clearest ways of stating the apparent need to cancel gauge anomalies in Yang-Mills theory with chiral fermions. However, we have seen through this specific example that such a metamorphosis of constraints can indeed happen — with important physical consequence. Before musing on the possible implications of this for gauge theories, we first need to show that this flexibility in the class of the constraints is generic; that is, we shall demonstrate that such a generalized Dirac approach is needed in order to recover Mackey's description of the inequivalent quantizations on a generic $\mathrm{G} / \mathrm{H}$. 


\section{Generalized Dirac approach to quantizing on coset spaces}

We have seen in the previous section that a constrained description of Mackey's account of spin can be developed which has many attractive features; not the least of which is the avoidence of vector-valued wave functions in the characterization of quantum states. The aim of this section is to extend that constrained analysis to the general coset space $\mathrm{G} / \mathrm{H}$ discussed by Mackey. We will clearly state the generalized version of Dirac's approach appropriate to this situation, and prove that the results so obtained agree with those proposed by Mackey for the different quantum sectors of these theories. Finally we shall investigate the group invariance of the physical states, and show that they are defined over a product of the coset space $\mathrm{G} / \mathrm{H}$ and a coadjoint orbit of $\mathrm{H}$.

\subsection{Hamiltonian dynamics on $\mathrm{G}$ and classical reduction to $\mathrm{G} / \mathrm{H}$}

The systems we wish to quantize are those describing free (geodesic) motion on the configuration space $Q \simeq \mathrm{G} / \mathrm{H}$, with respect to the metric $g_{\alpha \beta}$ induced from the Killing metric on $\mathrm{G}$. This dynamics, in turn, can be recovered from a reduction of the free motion on the extended configuration space G. Here we want to recapitulate how this reduction is described within the Hamiltonian formulation of dynamics.

The kinematical arena for the Hamiltonian description of dynamics on the Lie group $\mathrm{G}$ is the phase space $T^{*} \mathrm{G}[25]$. This cotangent bundle is actually a trivial bundle over $\mathrm{G}$ and can be identified with $\mathrm{G} \times \mathfrak{g}$. If $\left\{T_{m}\right\}$ is a basis of $\mathfrak{g}$ so that the point $R \in \mathfrak{g}$ can be written as $R=R^{m} T_{m}$, and $g^{-1} d g$ is the left-invariant Maurer-Cartan 1-form on $\mathrm{G}$, then the above trivialization of $T^{*} \mathrm{G}$ amounts to identifying $(g, R) \in \mathrm{G} \times \mathfrak{g}$ with $R_{m}\left(g^{-1} d g\right)^{m} \in T^{*} \mathrm{G}$, where $R_{m}:=\operatorname{Tr}\left(T_{m} R\right)$.

As with any cotangent bundle, this phase space comes equipped with a canonical symplectic 2-form $\omega$ from which the Poisson bracket between functions can be calculated. In terms of the above trivialization of $T^{*} \mathrm{G}$, this symplectic 2 -form is given by

$$
\omega=d \theta, \quad \text { where } \quad \theta:=-\operatorname{Tr} R\left(g^{-1} d g\right) .
$$

Using a matrix representation of the elements of $\mathrm{G}$ so that $g \in \mathrm{G}$ has matrix elements $g_{i j}$, this symplectic form generates the fundamental Poisson bracket (see Appendix B):

$$
\left\{g_{i j}, g_{k l}\right\}=0, \quad\left\{R_{m}, g_{i j}\right\}=\left(g T_{m}\right)_{i j}, \quad\left\{R_{m}, R_{n}\right\}=f_{m n}^{l} R_{l}
$$


Thus we see that the 'right-currents' $R_{m}$ generate the right action of G on itself, $g \rightarrow g \tilde{g}$, where $\tilde{g} \in \mathrm{G}$. There is also a left action of $\mathrm{G}$ on itself, $g \rightarrow \tilde{g}^{-1} g$, which is generated by the 'left-currents' $L_{m}$ given by

$$
L_{m}:=-\operatorname{Tr}\left(T_{m} g R g^{-1}\right)
$$

The left-currents satisfy the Poisson bracket

$$
\left\{L_{m}, g_{i j}\right\}=-\left(T_{m} g\right)_{i j}, \quad\left\{L_{m}, L_{n}\right\}=f_{m n}^{l} L_{l}
$$

which can be confirmed from (4.2) and (4.3). It is clear from the left and the right group actions that the left and right-currents commute,

$$
\left\{R_{m}, L_{n}\right\}=0
$$

We take as our Hamiltonian on $\mathrm{G}$

$$
H_{\mathrm{G}}=\frac{1}{2} \operatorname{Tr} R^{2}=\frac{1}{2} \eta^{m n} R_{m} R_{n}
$$

which can equally be written, from (4.3), as $H_{\mathrm{G}}=\frac{1}{2} \operatorname{Tr} L^{2}$. The equations of motion derived from (4.6) are then

$$
\frac{d}{d t}\left(g^{-1} \dot{g}\right)=0
$$

which decribe geodesic motion on G. To confirm this, we introduce a set of local coordinates $\left\{x^{\mu}\right\}(\mu=1, \ldots, \operatorname{dim} \mathrm{G})$ parametrizing $g=g(x)$, and recall that the natural metric on $\mathrm{G}$ is given by $g_{\mu \nu}:=\eta_{m n} v_{\mu}^{m} v_{\nu}^{n}$, where $v=v_{\mu}^{m} d x^{\mu} T_{m}:=g^{-1} d g$ is the vielbein 1 -form defined from the left invariant 1 -form. Then, using these, we can convert (4.7) into

$$
\ddot{x}^{\mu}+\Gamma_{\nu \lambda}^{\mu}(x) \dot{x}^{\nu} \dot{x}^{\lambda}=0,
$$

where $\Gamma_{\nu \lambda}^{\mu}$ is the Levi-Civita connection. An alternative and quicker way to arrive at the geodesic equation (4.8) is to derive the Lagrangian from (4.1) and (4.6) by Legendre transform,

$$
L_{\mathrm{G}}=\frac{1}{2} \operatorname{Tr}\left(g^{-1} \dot{g}\right)^{2}=\frac{1}{2} g_{\mu \nu}(x) \dot{x}^{\mu} \dot{x}^{\nu}
$$

where now (4.8) is obvious.

For the reduction $\mathrm{G} \rightarrow \mathrm{G} / \mathrm{H}$, we first note that the right action of the subgroup $\mathrm{H}$ on $\mathrm{G}$ is generated by the right currents $R_{i}=\operatorname{Tr}\left(T_{i} R\right)$, where $\left\{T_{i}\right\}$ is a basis of $\mathfrak{h}$ in the 
reductive decomposition $\mathfrak{g}=\mathfrak{h} \oplus \mathfrak{r}$. The currents $R_{i}$ form the algebra $\mathfrak{h}$ under Poisson bracket,

$$
\left\{R_{i}, R_{j}\right\}=f_{i j}^{k} R_{k}
$$

and can be used to implement the reduction from the extended phase space $T^{*} \mathrm{G}$ to the reduced phase space $T^{*}(\mathrm{G} / \mathrm{H})$, i.e, we take as our classical, first class constraints

$$
R_{i}=0
$$

The Hamiltonian (4.6) preserves these constraints, and on the constrainted surface given by (4.11) it reduces to the (physical) Hamiltonian,

$$
H_{\mathrm{G} / \mathrm{H}}=\left.H_{\mathrm{G}}\right|_{R_{i}=0}=\frac{1}{2} \eta^{a b} R_{a} R_{b}
$$

On the other hand, given a section $\sigma: \mathrm{G} / \mathrm{H} \mapsto \mathrm{G}$ we can write $g=\sigma h$ with $\sigma \in \mathrm{G}, h \in \mathrm{H}$. Using a set of local coordinates $\left\{q^{\alpha}\right\}$ on $\mathrm{G} / \mathrm{H}$ to parametrize $\sigma=\sigma(q)$, and performing Legendre transform we obtain the reduced Lagrangian

$$
L_{\mathrm{G} / \mathrm{H}}=\frac{1}{2} \operatorname{Tr}\left(\left.\sigma^{-1} \dot{\sigma}\right|_{\mathfrak{r}}\right)^{2}=\frac{1}{2} g_{\alpha \beta}(q) \dot{q}^{\alpha} \dot{q}^{\beta},
$$

where we have defined the vielbein $e=e_{\alpha}^{a} d q^{\alpha} T_{a}:=\left.\sigma^{-1} d \sigma\right|_{\mathfrak{r}}$ to provide the metric $g_{\alpha \beta}:=$ $\eta_{a b} e_{\alpha}^{a} e_{\beta}^{b}$ on $\mathrm{G} / \mathrm{H}$. Then again, it is obvious that the equations of motion derived in the reduced system are of the form,

$$
\ddot{q}^{\alpha}+\Gamma_{\beta \gamma}^{\alpha}(q) \dot{q}^{\beta} \dot{q}^{\gamma}=0
$$

where $\Gamma_{\beta \gamma}^{\alpha}$ is the Levi-Civita connection constructed out of the metric $g_{\alpha \beta}$. Thus we see that the dynamics of the reduced system is indeed that describing geodesic motion on $\mathrm{G} / \mathrm{H}$.

\subsection{Dirac's (naive) quantization on $\mathrm{G} / \mathrm{H}$}

The first step in any constrained description of quantizing on $\mathrm{G} / \mathrm{H}$ is to initially ignore the constraints (4.11) and quantize on the extended configuration space G. Any group $\mathrm{G}$ can be viewed trivially as a coset space $\mathrm{G} /\{1\}$, hence, using Mackey's approach discussed in Sect. 2, there is a well defined quantization in which the states are simply the square integrable wave functions $\psi(g)$ on $\mathrm{G}$ and the integration is with respect to the 
Haar-measure on $\mathrm{G}$. Then the representation (2.16) of $\mathrm{G}$ on these states is simply the left-regular representation

$$
\left(U_{\mathrm{L}}(\tilde{g}) \psi\right)(g)=\psi\left(\tilde{g}^{-1} g\right) .
$$

The generators of the left action are, from (2.22), simply the quantized left-currents $\widehat{L}_{m}$, and can be identified with ( $i$ times) the right-invariant vector fields on G. On top of this representation of $\mathrm{G}$, there is also the right-regular representation on these states

$$
\left(U_{\mathrm{R}}(\tilde{g}) \psi\right)(g)=\psi(g \tilde{g})
$$

which is clearly generated by the quantized right-currents $\widehat{R}_{m}$ and can be identified with ( $-i$ times) the left invariant vector fields on $\mathrm{G}$. Hence the commutators among the currents are

$$
\left[\widehat{R}_{m}, \widehat{R}_{n}\right]=i f_{m n}^{l} \widehat{R}_{l}, \quad\left[\widehat{L}_{m}, \widehat{L}_{n}\right]=i f_{m n}^{l} \widehat{L}_{l}, \quad\left[\widehat{R}_{m}, \widehat{L}_{n}\right]=0
$$

which are consistent with the Poisson bracket relations (4.2), (4.4) and (4.5).

Dirac's approach to the constrained quantization on $\mathrm{G} / \mathrm{H}$ identifies the physical (reduced) wave functions with those wave functions $\psi_{\text {phy }}$ on $\mathrm{G}$ which satisfy

$$
\widehat{R}_{i} \psi_{\text {phy }}(g)=0
$$

Such a condition on states is taken to be the natural translation of the classical condi$\operatorname{tion}^{5}(4.11)$. Thus, for such physical states we have

$$
\left(U_{\mathrm{R}}(h) \psi_{\text {phy }}\right)(g)=\psi_{\text {phy }}(g)
$$

for all $h \in \mathrm{H}$ (or more precisely, for all $h \in \mathrm{H}$ connected to the identity $h=1$ ). Then from (4.16) we find $\psi_{\text {phy }}(g)=\psi_{\text {phy }}(g H)$, which implies that the physical wave functions on $\mathrm{G}$ are, in effect, just wave functions on $\mathrm{G} / \mathrm{H}$. Since the left and right regular representations commute, we see that the left-regular represention (4.15) becomes the trivial representation on $L^{2}(\mathrm{G} / \mathrm{H})$ in Mackey's account of quantizing on $\mathrm{G} / \mathrm{H}$ (see (2.16)).

5 It should be noted, though, that in the classical theory gauge fixing is needed to directly isolate the physical states. It is also the case that in the path-integral account of this system one usually uses some gauge fixing. So it is a peculiar artifact of this system (based on the fact that $\mathrm{G}$ is compact so that there are normalisable solutions to (4.18) on $\left.L^{2}(G)\right)$ that gauge fixing appears to be inessential in the operator formalism. However, a more systematic approach [23] shows that gauge fixing has some significance even in this case. 
On the extended state space the quantized Hamiltonian (4.6) is given by

$$
\widehat{H}_{\mathrm{G}}=\frac{1}{2} \operatorname{Tr} \widehat{R}^{2}=\frac{1}{2} \eta^{m n} \widehat{R}_{m} \widehat{R}_{n}
$$

which on the physical states (4.18) reduces to the quantized version of the Hamiltonian $(4.12)$,

$$
\widehat{H}_{\mathrm{G} / \mathrm{H}}=\frac{1}{2} \eta^{a b} \widehat{R}_{a} \widehat{R}_{b}
$$

This is precisely the Laplace-Beltrami operator $-\frac{1}{2} \triangle_{\mathrm{LB}}$ acting on the wave function on $\mathrm{G} / \mathrm{H}$.

\subsection{Generalized Dirac quantization on $\mathrm{G} / \mathrm{H}$}

We have seen that Dirac's (naive) approach to quantization applied to the coset space $\mathrm{G} / \mathrm{H}$ recoveres only the trivial sector in Mackey's account of the inequivalent quantizations possible on such a space. Thus, following the philosophy outlined in the Introduction (as summarized in Fig. 2 there) we need to generalize Dirac's reduction prescription (4.18) so that all quantizations are recovered. From our analysis of Sect.3, we expect this to be achieved by putting the constraints $\widehat{R}_{i}$ equal to some constants, rather than zero as the classical theory might suggest. Following our discussion of Mackey, we expect these constants to be related to the irreducible representaions of $\mathrm{H}$. To start this generalization of Dirac's approach, though, we first need the following technical result that allows us to disentangle the $\mathrm{H}$ and $\mathrm{G} / \mathrm{H}$ parts of the wave functions on $\mathrm{G}$ :

$$
L^{2}(\mathrm{G}) \simeq L^{2}(\mathrm{G} / \mathrm{H} \times \mathrm{H}) \simeq L^{2}(\mathrm{G} / \mathrm{H}) \otimes L^{2}(\mathrm{H}) .
$$

This is, in fact, almost obvious if we recall that any fibre bundle is locally trivial, and can be written as a product of the base space and the fibre. For a formal proof, let us choose a section $\sigma$ and define the unitary mappings $T: L^{2}(\mathrm{G}) \mapsto L^{2}(\mathrm{G} / \mathrm{H} \times \mathrm{H})$ and $T^{*}: L^{2}(\mathrm{G} / \mathrm{H} \times \mathrm{H}) \mapsto L^{2}(\mathrm{G})$ by

$$
(T \psi)(q, h)=\psi(\sigma(q) h), \quad\left(T^{*} \psi\right)(g)=\psi\left(\operatorname{pr}(g), \sigma^{-1}(\operatorname{pr}(g)) g\right)
$$

where $\operatorname{pr}(g)$ is the projection $\mathrm{G} \mapsto \mathrm{G} / \mathrm{H}$ (see Sect.2.1). Then it is easy to see that these mappings are the inverse to each other; for example, we have

$$
\begin{aligned}
\left(T T^{*} \psi\right)(q, h) & =\left(T^{*} \psi\right)(\sigma(q) h) \\
& =\psi\left(\operatorname{pr}(\sigma(q) h), \sigma^{-1}(\operatorname{pr}(\sigma(q) h)) \sigma(q) h\right) \\
& =\psi(q, h)
\end{aligned}
$$


and the other way around is even easier. The unitarity of these mappings follows from the definition (2.12) of the invariant measure on $\mathrm{G} / \mathrm{H}$. On account of this isomorphism, in what follows we sometimes write $\psi(g)$ as $\psi(q, h)$ or vise versa by abuse of notation.

On $L^{2}(\mathrm{G} / \mathrm{H} \times \mathrm{H})$ the left-regular representation (4.15) becomes

$$
\left(U_{\mathrm{L}}(g) \psi\right)(q, h)=\psi\left(g^{-1} q, h_{\sigma}\left(g^{-1}, q\right) h\right),
$$

where $h_{\sigma}\left(g^{-1}, q\right)$ is the element of $\mathrm{H}$ defined in (2.9). Given this decomposition of $L^{2}(\mathrm{G})$ we can further decompose the wave functions on $\mathrm{H}$ by using the Peter-Weyl theorem. Recall that given an irreducible representation of $\mathrm{H}$, labelled by the heighest weight $\chi$, we introduce a basis of states $|\chi, \mu\rangle$ and write the matrix elements of this representation as $\pi_{\mu \nu}^{\chi}(h)$ as in (2.17). Then we have the orthogonality relations

$$
\int_{\mathrm{H}} D h \pi_{\mu \mu^{\prime}}^{\chi^{*}}(h) \pi_{\nu \nu^{\prime}}^{\chi}(h)=\frac{1}{d_{\chi}} \delta_{\mu \nu} \delta_{\mu^{\prime} \nu^{\prime}},
$$

where $d_{\chi}$ is the dimension of the representation $\chi$, and the Peter-Weyl theorem tells us that we can write any wave function $\psi(h)$ on $\mathrm{H}$ as

$$
\psi(h)=\sum_{\chi, \mu, \mu^{\prime}} C_{\mu \mu^{\prime}}^{\chi} \pi_{\mu \mu^{\prime}}^{\chi *}(h)
$$

Combining this result with the isomorphism (4.22), we see that any wave function $\psi(g)$ on G can be written as

$$
\psi(g)=\sum_{\chi, \mu, \mu^{\prime}} \psi_{\mu \mu^{\prime}}^{\chi}(q) \pi_{\mu \mu^{\prime}}^{\chi *}(h)
$$

where now the coefficients $\psi_{\mu \mu^{\prime}}^{\chi}(q)$ are wave functions on $\mathrm{G} / \mathrm{H}$.

Now let $K$ be an element of the Lie algebra $\mathfrak{h}$ and consider the operators

$$
\widehat{\phi}_{i}=\operatorname{Tr} T_{i}(\widehat{R}-K)=\widehat{R}_{i}-K_{i}
$$

We identify these with the new, effective constraints of the quantum theory. However, the functions $\widehat{\phi}_{i}$ represent a mixed set (first and second class) and thus cannot be directly used to define the physical states. Indeed, from (4.17) the commutator of these operators are

$$
\left[\widehat{\phi}_{i}, \widehat{\phi}_{j}\right]=i f_{i j}^{k} \widehat{\phi}_{k}+i \operatorname{Tr}\left(\left[T_{i}, T_{j}\right] K\right)
$$


and hence the $\operatorname{Tr}\left(\left[T_{i}, T_{j}\right] K\right)$ term signals the existence of some second class components in the set (4.29). In order to isolate the first class subset of (4.29) we consider the subalgebra $\mathfrak{s}_{K}$ given by the kernel of the adjoint action of $K$ in $\mathfrak{h}$,

$$
\mathfrak{s}_{K}:=\operatorname{Ker}\left(\operatorname{ad}_{K}\right) \cap \mathfrak{h} .
$$

For a generic $K$, that is, if $K$ is a regular semisimple element in $\mathfrak{h}$, the subalgebra $\mathfrak{s}_{K}$ is precisely the Cartan subalgebra $\mathfrak{t}$ of $\mathfrak{h}$ containing $K$ [26]. If not, $\mathfrak{s}_{K}$ is larger than $\mathfrak{t}$ and, due to the non-degeneracy of $\mathfrak{t}$ with respect to the Killing form, admits the decomposition $\mathfrak{s}_{K}=\mathfrak{t} \oplus \mathfrak{c}$ where $\mathfrak{c}$ is the orthogonal complement. Choosing a basis $\left\{T_{s}\right\}$ in $\mathfrak{s}_{K}$ (we shall use $\left\{T_{r}\right\}$ and $\left\{T_{p}\right\}$ for the bases in $\mathfrak{t}$ and $\mathfrak{c}$, respectively), we see that for any $T_{j} \in \mathfrak{h}$ we have $\operatorname{Tr}\left(\left[T_{s}, T_{j}\right] K\right)=0$ and hence the first class components in (4.29) are given by

$$
\widehat{\phi}_{s}:=\operatorname{Tr} T_{s}(\widehat{R}-K) \text {. }
$$

Conversely, from the semisimplicity of $\mathfrak{h}$ it follows that these $\widehat{\phi}_{s}$ form the maximal set of the first class components in (4.29).

Just as we did for the spin example in Sect.3, we now need to find a 'maximal first class subalgebra' formed by the operators in (4.29), which involves working with the complex extension $\mathfrak{h}_{\mathrm{c}}$ of the algebra $\mathfrak{h}$. Introducing a Chevalley basis $\left(H_{\alpha_{r}}, E_{ \pm \varphi}\right)$ in $\mathfrak{h}_{\mathrm{c}}$ (see Appendix A) where we take $T_{r}:=\frac{1}{i} H_{\alpha_{r}}(r=1, \ldots$, rank H), we see that a maximal first class subalgebra of $(4.29)$ is given by the set $\left(\widehat{\phi}_{s}, \widehat{\phi}_{\varphi}\right)$, where we have defined

$$
\widehat{\phi}_{\varphi}=\operatorname{Tr} E_{-\varphi}(\widehat{R}-K)
$$

for all positive roots $\varphi$. If $K_{r}=\operatorname{Tr}\left(T_{r} K\right)$ has integer values corresponding to the heighest weight $\chi$, i.e., $K_{r}=\chi\left(H_{\alpha_{r}}\right)$, then the physical states defined by the conditions

$$
\widehat{\phi}_{s} \psi_{\text {phy }}(g)=0 \quad \text { and } \quad \widehat{\phi}_{\varphi} \psi_{\text {phy }}(g)=0
$$

have the solutions of the form

$$
\psi_{\text {phy }}(g)=\psi_{\text {phy }}(q, h)=\sum_{\mu} \psi_{\mu}(q) \pi_{\mu \chi}^{\chi *}(h)
$$

For the generic case where $K$ is regular semisimple the coefficient functions $\psi_{\mu}(q)$ are completely arbitrary, while for non-generic $K$ they must satisfy certain conditions so that 
the physical wave functions (4.35) be invariant under the transformations generated by $\widehat{\phi}_{p}$. In any case the wave functions (4.35) provide the correct identification with the physical states corresponding to the quantization on $\mathrm{G} / \mathrm{H}$ labelled by $\chi$ in the sense that the coefficient wave functions in (4.35) are precisely the vector-valued wave functions (2.14) used in Mackey's analysis. Indeed, given a physical wave function (4.35), the coefficient functions in (4.35) are obtained as

$$
\psi_{\mu}(q)=d_{\chi} \int_{\mathrm{H}} D h \psi_{\mathrm{phy}}(q, h) \pi_{\mu \chi}^{\chi}(h)
$$

Hence under the left action (4.25) we have

$$
\begin{aligned}
\psi_{\mu}(q) & \rightarrow d_{\chi} \int_{\mathrm{H}} D h \psi_{\mathrm{phy}}\left(g^{-1} q, h_{\sigma}\left(g^{-1}, q\right) h\right) \pi_{\mu \chi}^{\chi}(h) \\
& =d_{\chi} \int_{\mathrm{H}} D h \sum_{\nu} \psi_{\nu}\left(g^{-1} q\right) \pi_{\nu \chi}^{\chi^{*}}\left(h_{\sigma}\left(g^{-1}, q\right) h\right) \pi_{\mu \chi}^{\chi}(h) \\
& =d_{\chi} \sum_{\mu, \nu} \psi_{\nu}\left(g^{-1} q\right) \pi_{\nu \lambda}^{\chi *}\left(h_{\sigma}\left(g^{-1}, q\right)\right) \int_{\mathrm{H}} D h \pi_{\lambda \chi}^{\chi *}(h) \pi_{\mu \chi}^{\chi}(h) \\
& =\sum_{\nu} \pi_{\mu \nu}^{\chi}\left(h_{\sigma}\left(g, g^{-1} q\right)\right) \psi_{\nu}\left(g^{-1} q\right) .
\end{aligned}
$$

Thus we see that the left-regular representation (4.25) reproduces exactly the induced representation (2.16), and hence $\psi_{\mu}(q)$ are identified with the vector-valued wave functions used by Mackey. This proves the equivalence between our generalized Dirac approach and Mackey's approach, and the formulae, (4.35) and (4.36), provide the mapping between the two.

In the trivial sector we have seen from (4.19) that the physical wave functions on $G$ can be identified with the wave functions on the physical (classical) configuration space $\mathrm{G} / \mathrm{H}$. In the remainder of this section we shall investigate which space the wave functions (4.35) are naturally defined over when we are not in the trivial sector.

The first thing to note is that the physical states (4.35) are not $\mathrm{H}$-invariant. In fact, using the identification (4.22), the right action of $\mathrm{H}$ on $L^{2}(\mathrm{G})$ is simply

$$
\left(U_{\mathrm{R}}(\tilde{h}) \psi\right)(q, h)=\psi(q, h \tilde{h}) .
$$

So acting on a physical state (4.35) we get

$$
\begin{aligned}
\left(U_{\mathrm{R}}(\tilde{h}) \psi_{\text {phy }}\right)(q, h) & =\psi_{\text {phy }}(q, h \tilde{h}) \\
& =\sum_{\mu} \psi_{\mu}(q) \pi_{\mu \nu}^{\chi^{*}}(h) \pi_{\nu \chi}^{\chi^{*}}(\tilde{h}),
\end{aligned}
$$


which, for a general $\tilde{h} \in \mathrm{H}$, is not related in any obvious way to the original physical state. However, if $\tilde{h}=s \in \mathrm{S}_{K}$ where $\mathrm{S}_{K}$ is the subgroup of $\mathrm{H}$ obtained by the exponential mapping of the subalgebra $\mathfrak{s}_{K}$ in (4.31), then we can write $s=e^{T_{r} \theta^{r}} e^{T_{p} \theta^{p}}$, whereby (4.39) becomes

$$
\psi_{\text {phy }}(q, h s)=e^{i K_{r} \theta^{r}} \psi_{\text {phy }}(q, h) .
$$

From (4.38) we see that this $\mathrm{S}_{K}$-invariance of the physical states only affects the $\mathrm{H}$ factor in the decomposition $L^{2}(\mathrm{G}) \simeq L^{2}(\mathrm{G} / \mathrm{H} \times \mathrm{H})$. Indeed, just as we did for wave functions on $\mathrm{G}$, we can decompose further $L^{2}(\mathrm{H})$ as

$$
L^{2}(\mathrm{H}) \simeq L^{2}\left(O_{K} \times \mathrm{S}_{K}\right)
$$

where $O_{K}:=\mathrm{H} / \mathrm{S}_{K}$ is the coadjoint orbit through $K \in \mathfrak{h}$. Clearly, due to the $\mathrm{S}_{K^{-}}$ invariance the physical wave functions on $\mathrm{G}$ can be identified with wave functions on the (effective) physical space $\mathrm{G} / \mathrm{H} \times O_{K}$. More precisely, these wave functions are associated with quantizing on the classical phase space $T^{*}(\mathrm{G} / \mathrm{H}) \times O_{K}$ - they are not the whole of $L^{2}\left(\mathrm{G} / \mathrm{H} \times O_{K}\right)$. Thus we see that, in addition to the degrees of freedom on $\mathrm{G} / \mathrm{H}$ that we naively expect, we find extra degrees of freedom represented by the coadjoint orbit $O_{K}$. In the example we discussed in Sect. 3, these were in fact spin degrees of freedom, and by this reasoning they will be henthforth called 'generalized spin' in the general case.

The loss of the H-invariance, though, on the extended state space is not too attractive when we try and generalize these ideas to field theory where we still want to have gauge invariance. We may, however, restore the $\mathrm{H}$-invariance at the expense of working on a larger state space. This can easily be accomplished by constructing new wave functions defined on $\mathrm{G} \times \mathrm{H}$ from the physical wave functions in (4.35) by

$$
\Psi(g, h):=\psi_{\text {phy }}(g h)=\sum_{\mu} \Psi_{\mu}(g) \pi_{\mu \chi}^{\chi *}(h),
$$

where the coefficient wave functions on $\mathrm{G}$ are given by

$$
\Psi_{\mu}(g):=\sum_{\nu} \psi_{\nu}(\operatorname{pr}(g)) \pi_{\nu \mu}^{\chi *}\left(\sigma^{-1}(\operatorname{pr}(g)) g\right) .
$$

Clearly, $\Psi(g, h)$ has the (trivial) H-invariance as well as the $\mathrm{S}_{K}$-invariance,

$$
\Psi\left(g \tilde{h}, \tilde{h}^{-1} h\right)=\Psi(g, h), \quad \Psi(g, h s)=e^{i K_{r} \theta^{r}} \Psi(g, h) .
$$

Note that the coefficient wave functions (4.43) satisfy the identity

$$
\Psi_{\mu}(g \tilde{h})=\sum_{\nu} \pi_{\mu \nu}^{\chi}\left(\tilde{h}^{-1}\right) \Psi_{\nu}(g) .
$$

Conversely, given a collection of wave functions $\Psi_{\mu}(g)$ on $\mathrm{G}$ which satisfies (4.45), then they can be written in the form (4.43). In fact these are the form of the vector-valued wave functions used by Mackey [6]. 


\section{Path-integral description for quantizing on $\mathrm{G} / \mathrm{H}$}

In the previous section we have presented a generalization of Dirac's approach to constrained quantization, in order to deal with inequivalent quantizations on a coset space $\mathrm{G} / \mathrm{H}$. In this section we develop the corresponding language in the path-integral which renders the treatise more transparent and admits a more intuitive understanding of the inequivalent quantizations. In particular, we shall see that in the path-integral the appearance of the generalized spin discussed in Sect. 4 is realized immediately, and that the emergence of the H-connection is also trivial to observe. Furthermore, the consistency of the path-integral under the $\mathrm{S}_{K}$-transformations requires the parameters in $K$ to be precisely those labelling the irreducible representations $\chi$ of $\mathrm{H}$. The equations of motion on $\mathrm{G} / \mathrm{H}$ turn out to be of the type of the Wong equations [27], describing a particle minimally coupled to the H-connection and the generalized spin.

\subsection{Lagrangian realization in the path-integral}

We have seen, in Sect. 4, that the inequivalent quantizations on the coset space $\mathrm{G} / \mathrm{H}$ can be derived from a generalized form of Dirac's constrained quantization applied to the classical system defined on $\mathrm{G}$, subject to the (classical) first class constraints $R_{i}=0$. This account has been an operator one. To develop the corresponding path-integral version we will simply extract from that analysis the fact that in the quantum theory these classical constraints become the effective, anomalous constraints

$$
\phi_{i}=\operatorname{Tr} T_{i}(R-K)=R_{i}-K_{i}=0,
$$

where $T_{i} \in \mathfrak{h}$ (for our notational conventions, see Appendix A), and then use the familiar prescriptions for implementing these within a path-integral [22]. At this stage the $K_{i}$ are only assumed to be arbitrary constants.

Due to the existence of a first class subset of these constraints (5.1) given by

$$
\phi_{s}=\operatorname{Tr} T_{s}(R-K)=0,
$$

for $T_{s} \in \mathfrak{s}_{K}=\operatorname{Ker}\left(\operatorname{ad}_{K}\right) \cap \mathfrak{h}$, the resulting theory acquires a gauge symmetry under the $\mathrm{S}_{K^{-}}$ transformations associated with the algebra $\mathfrak{s}_{K}$. Accordingly, we introduce gauge fixing 
conditions $\xi_{s}=0$ so that the total set of constraints $\varphi_{k}:=\left(\phi_{i}, \xi_{s}\right)$ is second class. With this second class set of constraints the phase space path-integral reads

$$
Z=\int \mathcal{D} g \mathcal{D} R \delta\left(\varphi_{k}\right) \operatorname{det}^{\frac{1}{2}}\left|\left\{\varphi_{k}, \varphi_{k^{\prime}}\right\}\right| \exp \left(i \int \theta-i \int_{0}^{T} d t H_{\mathrm{G}}\right)
$$

where $\theta$ is the canonical 1 -form in $(4.1)$,

$$
\int \theta=-\int_{0}^{T} d t R_{m}\left(g^{-1} \dot{g}\right)^{m}
$$

and $H_{\mathrm{G}}$ is the Hamiltonian (4.6). The path-integral measure in (5.3) is formally defined from the volume (Liouville) form of the phase space $\omega^{N}(N=\operatorname{dim} \mathrm{G})$ by taking its product over $t$,

$$
\mathcal{D} g \mathcal{D} R=\prod_{t} \omega^{N}(t), \quad \text { where } \quad \omega^{N}=\prod_{m=1}^{N}\left(g^{-1} d g\right)^{m} d R_{m}
$$

On account of the fact that the operators in (4.34) form the maximal first class subalgebra of (4.29), the operator version of (5.1), it is clear that the constrained path-integral (5.3) leads to the quantum theory equivalent to that obtained by the operator description of Sect. 4 .

Since the constraints (5.1) are (at most) linear in the momentum variables $R_{i}$, we may trivially implement them by integrating over all the momentum variables $R_{m}$. For this, we first note that the determinant factor in (5.3) is proportional to $\operatorname{det}\left|\left\{\phi_{s}, \xi_{s^{\prime}}\right\}\right|$ on the constrained surface. Thus, if we choose the gauge fixing conditions $\xi_{s}=0$ so that the determinant be independent of $R_{m}$, we can at once carry out the integrations on $R_{m}$ to obtain

$$
Z=\int \mathcal{D} g \delta\left(\xi_{s}\right) \operatorname{det}\left|\left\{\phi_{s}, \xi_{s^{\prime}}\right\}\right| \exp \left(i \int_{0}^{T} d t L_{\text {tot }}\right)
$$

where

$$
L_{\text {tot }}=\frac{1}{2} \operatorname{Tr}\left(\left.g^{-1} \dot{g}\right|_{\mathfrak{r}}\right)^{2}-\operatorname{Tr} K\left(\left.g^{-1} \dot{g}\right|_{\mathfrak{h}}\right)
$$

Note that since the first term in the Lagrangian (5.7) is invariant under $g \rightarrow g \tilde{h}$ for $\tilde{h} \in \mathrm{H}$ (which follows from the identity $\left.h^{-1} X h\right|_{\mathfrak{r}}=\left.h^{-1} X\right|_{\mathfrak{r}} h$ valid for any $X \in \mathfrak{g}$ as a consequence of the reductive decomposition), it is actually identical to the Lagrangian (4.13) for $\mathrm{G} / \mathrm{H}$. On the other hand, it will be shown that the second term in the Lagrangian (5.7) represents the effects of non-trivial quantizations, containing the H-connection and the generalized spin. In passing we point out that the path-integral (5.6) is not quite a configuration 
path-integral due to the second term which is first order in time derivative. We also note that the measure $\mathcal{D} g=\prod_{t, m}\left(g^{-1} d g\right)^{m}(t)$ in (5.6) is formally the product of the Haar measure of the group $\mathrm{G}$ over $t$, and by this expression it is meant to give the sum over all possible paths allowed by the prescribed boundary condition (to be discussed later), which includes 'multi-winding' paths going around several times the fundamental region of the group manifold.

\subsection{Parameter quantization of $K$}

We know, from Sect. 4 , that the parameters in $K$ are not quite arbitrary; they must be those (integers) that label the highest weight representation $\chi$ of $\mathrm{H}$ on which the Hilbert space is constructed. We now show that this particular information has already been incorporated in our path-integral. To this end, we first observe that, under the $\mathrm{S}_{K}$-transformations $g \rightarrow g s$ for $s \in \mathrm{S}_{K}$ generated by the first class set (5.2), the total Lagrangian varies as

$$
L_{\text {tot }} \longrightarrow L_{\text {tot }}+\Delta L_{\text {tot }}, \quad \text { where } \quad \Delta L_{\text {tot }}=-\operatorname{Tr} K\left(s^{-1} \dot{s}\right)
$$

As in the previous section, if we use the parametrization $s=e^{\theta^{r} T_{r}} e^{\xi^{p} T_{p}}$ where $\left\{T_{r}\right\}$ and $\left\{T_{p}\right\}$ are bases in $\mathfrak{t}$ and $\mathfrak{c}$ in the orthogonal decomposition $\mathfrak{s}_{K}=\mathfrak{t} \oplus \mathfrak{c}$, then

$$
\Delta L_{\mathrm{tot}}=-\frac{d}{d t}\left(K_{r} \theta^{r}\right)
$$

Thus the Lagrangian is invariant up to a total time derivative, implying that the equations of motion are invariant under the $\mathrm{S}_{K}$-transformation, which is the $\mathrm{S}_{K}$-symmetry at the classical level.

However, if we require the $\mathrm{S}_{K}$-symmetry to persist at the quantum level (which we must to ensure that the path-integral (5.6) is independent of the gauge fixing), then we need to take into account the boundary effect in the path-integral. To examine this explicitly, consider the transition amplitude from an initial point $g_{0}$ at $t=0$ to a final point $g_{1}$ at $t=T$. The sum in the path-integral contains all possible paths $g(t)$ going from $g(0)=g_{0}$ to $g(T)=g_{1}$, but to each such path there is a class of paths related each other by a gauge transformation,

$$
g(t) \longrightarrow g(t) s(t), \quad \text { with } \quad s(0)=s(T)=1
$$


The gauge invariance at the quantum level requires that the paths within a gauge equivalent class must contribute to the sum of the path-integral with the same amplitude, i.e., they must have the same phase factor. Using the Chevalley basis for our basis $T_{r}=\frac{1}{i} H_{\alpha_{r}}$, we find from the periodic property (see Appendix A) that gauge transformations satisfying the condition (5.10) are of the type,

$$
s(t)=e^{\theta^{r}(t) T_{r}}, \quad \text { with } \quad \theta^{r}(T)-\theta^{r}(0)=2 \pi n_{r},
$$

where $n_{r}$ are integers ${ }^{6}$. From this one sees that the requirement $e^{i \int_{0}^{T} d t \Delta L_{\text {tot }}}=1$, or

$$
\int_{0}^{T} d t \Delta L_{\text {tot }}=-2 \pi n_{r} K_{r}=2 \pi \times \text { integer }
$$

for any class of gauge transformations $\left(i . e\right.$. , for any $\left.n_{r}\right)$ is equivalent to

$$
K_{r} \in \mathbb{Z}, \quad \text { for } \quad r=1, \ldots, \operatorname{rank} \mathrm{H}
$$

These integer parameters correspond precisely to the ones which label the highest weight representations of $\mathrm{H}$, with the identification being $K_{r}=\chi\left(H_{\alpha_{r}}\right)$, and this parameter quantization of $K$ completes the bridge between the operator description of Sect. 4 and the path-integral description given here. Accordingly, we see that our simple implementation of the generalized Dirac approach in the path-integral, where one just replaces the naive constraints (4.11) with (5.1), results in the same quantizations as those obtained in Mackey's approach which is based on the system of imprimitivity on $\mathrm{G} / \mathrm{H}$.

\subsection{Emergence of the generalized spin and the H-connection}

We now examine the dynamical implications of the Lagrangian (5.7). In particular, we are interested in the effects which arise when non-trivial quantizations are adopted for quantizing on the coset space $\mathrm{G} / \mathrm{H}$. From the identification that the constant $K$ labels the representation $\chi$, we expect that those effects take place with the 'strength' determined by $K$.

6 More precisely, $n_{r}$ are integers for the universal covering group $\widetilde{\mathrm{H}}$ of $\mathrm{H}$. For a nonsimply connected group $\mathrm{H}$, they are multiple of integers determined by the discrete normal subgroup $\mathrm{N}$ of $\widetilde{\mathrm{H}}$ for which $\mathrm{H} \simeq \widetilde{\mathrm{H}} / \mathrm{N}$. For instance, for $\mathrm{SU}(2)$ they are integers but for $\mathrm{SO}(3) \simeq \mathrm{SU}(2) / \mathbb{Z}_{2}$ they are even integers. Thus for non-simply connected groups the quantization condition for $K_{r}$ should be modified accordingly. 
Let us start by decomposing $g$ as

$$
g=\sigma h, \quad \text { with } \quad \sigma \in \mathrm{G}, \quad h \in \mathrm{H},
$$

where $\sigma=\sigma(q)$ is a section $\mathrm{G} / \mathrm{H} \mapsto \mathrm{G}$, and $\left\{q^{\alpha}\right\}$ is a set of local coordinates on $\mathrm{G} / \mathrm{H}$. Then the Lagrangian (5.7) becomes the sum of three terms:

$$
\begin{aligned}
L_{\mathrm{tot}} & =L_{\mathrm{G} / \mathrm{H}}+L_{O_{K}}+L_{\mathrm{int}} \\
& =\frac{1}{2} g_{\alpha \beta}(q) \dot{q}^{\alpha} \dot{q}^{\beta}-\operatorname{Tr} K\left(h^{-1} \dot{h}\right)-\operatorname{Tr}\left(h K h^{-1} A_{\alpha}(q)\right) \dot{q}^{\alpha} .
\end{aligned}
$$

Besides the first term in (5.15), which is the Lagrangian $L_{\mathrm{G} / \mathrm{H}}$ (4.13) for a free particle moving on $\mathrm{G} / \mathrm{H}$, we now recognize two terms which represent the effects of non-trivial quantizations and are both proportional to $K$. The effects are two-fold: first, there appears extra degrees of freedom $h \in \mathrm{H}$ which, as we shall see in the next section, correspond to the generalized spin. Their dynamics is governed by $L_{O_{K}}$, which is the first order Lagrangian for the coadjoint orbit $O_{K}=\mathrm{H} / \mathrm{S}_{K}$ of the group $\mathrm{H}$ passing through $K$. (Note that the Lagrangian $L_{O_{K}}$ is invariant under the $\mathrm{S}_{K}$-transformations and hence the phase space for the generalized spin is given by $\mathrm{H} / \mathrm{S}_{K}$.) The second effect appears in the last term $L_{\text {int }}$, where one finds the $\mathrm{H}$-connection

$$
A:=\left.\sigma^{-1} d \sigma\right|_{\mathfrak{h}}=A_{\alpha} d q^{\alpha}
$$

coupled minimally to the particle and the generalized spin. Note that if $\mathrm{H}$ is abelian then the coadjoint orbits $O_{K}$ are just points and therefore there appears no generalized spin. If, on the other hand, $\mathrm{H}=\mathrm{G}$ then there emerges no $\mathrm{H}$-connection; only the generalized spin could exist. The spin example discussed in Sect. 3 falls into the latter case (where $\mathrm{G} / \mathrm{H}=\mathrm{SU}(2) / \mathrm{SU}(2))$, while the example of the quantization on $S^{2}$ to be discussed in the next section is in the former case (where $\mathrm{G} / \mathrm{H}=\mathrm{SO}(3) / \mathrm{SO}(2)$ ).

The effects of the inequivalent quantizations in the dynamics can be seen in the equations of motion. In terms of the $\left(\mathrm{S}_{K}\right.$-gauge invariant) variables

$$
S:=-h K h^{-1}
$$

the equations of motion for $h$ derived from the Lagrangian (5.15) are the covariant constancy equations,

$$
D_{t} S:=\frac{d S}{d t}+\left[A_{\alpha}(q), S\right] \dot{q}^{\alpha}=0
$$


Whereas, for the particle motion we get

$$
\ddot{q}^{\alpha}+\Gamma_{\beta \gamma}^{\alpha}(q) \dot{q}^{\beta} \dot{q}^{\gamma}-g^{\alpha \beta}(q) S_{i} F_{\beta \gamma}^{i}(q) \dot{q}^{\gamma}=0
$$

where $F:=d A+A \wedge A$ is the curvature 2-form. Eqs. (5.18) and (5.19) are essentially the Wong equations [27] under the special, background non-abelian potential, the Hconnection, with the couplings (the parameters in $K$ ) in $S$ taking only discrete values (they are quantized).

\subsection{H-gauge structure}

The Wong equations were originally designed to provide a gauge invariant minimal coupling with a non-abelian potential, as a generalization of the minimal coupling with an electromagnetic potential. The same is true for our Lagrangian (5.15); it is invariant under the H-gauge transformation,

$$
A \longrightarrow \tilde{h}^{-1} A \tilde{h}+\tilde{h}^{-1} d \tilde{h}
$$

which is induced by the change of section,

$$
\sigma \longrightarrow \sigma \tilde{h}, \quad h \longrightarrow \tilde{h}^{-1} h, \quad \text { for } \quad \tilde{h} \in \mathrm{H}
$$

This H-gauge invariance is, of course, trivial since it derives from the decomposition (5.14). Interestingly, however, it can be made non-trivial when $K \neq 0$, i.e., when a non-trivial quantization is considered. To see this, let us first note that the H-gauge invariance can still be observed even after we fix the $S_{K}$-gauge invariance. Indeed, since gauge fixing for the $\mathrm{S}_{K}$-invariance is achieved simply by decomposing $h$ using a section $\tau: \mathrm{H} / \mathrm{S}_{K} \mapsto \mathrm{H}$,

$$
h=\tau s, \quad \text { with } \quad \tau \in \mathrm{H}, \quad s \in \mathrm{S}_{K},
$$

and setting $s=1$ subsequently, the change of section can still be performed by the slightly modified transformation,

$$
\sigma \longrightarrow \sigma \tilde{h}, \quad \tau \longrightarrow \tilde{h}^{-1} \tau \tilde{s}, \quad \text { for } \quad \tilde{h} \in \mathrm{H}
$$

where we choose $\tilde{s}=\tilde{s}(\tau, \tilde{h}) \in \mathrm{S}_{K}$ (see (2.9)) so that the transformation preserves the section $\tau$. But since the transformation (5.23) amounts just to the $\mathrm{S}_{K}$-transformation 
$g \rightarrow g \tilde{s}$, it leaves the path-integral (5.6) invariant thanks to the paramter quantization of $K$, hence the claimed H-gauge invariance.

An important point to note is that this transformation (5.23) does not involve a coordinate transformation for $q$ (since $\sigma(q) \rightarrow \sigma^{\prime}(q)=\sigma(q) \tilde{h}(q)$, see Sect. 2.1), and thus it can be interepreted as an ordinary gauge transformation for the background potential (5.20) and the generalized spin with $q$ fixed, under which the total Lagrangian is invariant up to a total time derivative. (Note that this is a non-trivial gauge transformation only for $K \neq 0$.) In fact, the $\mathrm{H}$-gauge structure we have just observed is a non-abelian, quantum mechanical version of the abelian, functional gauge structure found by Wu and Zee [28]. This suggests that when our approach is applied to Yang-Mills theory topological terms, and additional degrees of freedom, can also arise there from the inequivalent quantizations (for more detail, see [11]). Another point worth mentioning is that the type of Lagrangian (5.15) has been discussed by Balachandran et al. [29] in an effort to incorporate a Dirac monopole, non-relativistic spinning particle and the Wong equations in gauge invariant formulations. The discussion above shows that they all appear in the universal framework in our approach based on the reduction $\mathrm{G} \rightarrow \mathrm{G} / \mathrm{H}$.

We finish this section by noting the relation between the singularity/topology of the $\mathrm{H}$-connection and the $\mathrm{S}_{K}$-gauge invariance. Recall that a section $\sigma=\sigma(q)$ can be taken only at the cost of introducing some singularity in $q$ unless $\mathrm{G}$ is a direct product of $\mathrm{G} / \mathrm{H}$ and $\mathrm{H}$, i.e., unless the principal fibre bundle $\mathrm{G}$ is trivial. This means that in a generic situation the H-connection is singular, and it could be topologically non-trivial. Clearly, the locations of the singularity depend on the choice of the section, and can be moved freely if a change of section (5.21) is allowed. As we have discussed above, this is guaranteed by the $\mathrm{S}_{K}$-symmetry at the quantum level, which is ensured by the parameter quantization of $K$. However, it must be stressed that the parameter quantization of $K$ occurs irrespective of the non-triviality of the principal bundle $\mathrm{G}$ - it arises even when the bundle is trivial and the H-connection is perfectly regular on G/H. Such an example will be seen in the next section when we consider the quantization on $S^{3}$. 


\section{Physical implications of the inequivalant quantizations on $S^{n}$}

In this section we shall study the physical implications of inequivalent quantizations by quantizing on spheres $S^{n}$, regarding them as the coset spaces $S^{n} \simeq \mathrm{SO}(n+1) / \mathrm{SO}(n)$. As we have seen in the previous sections, the hallmark of inequivalent quantizations is the appearance of the $\mathrm{H}$-connection and the generalized spin given by a coadjoint orbit of $\mathrm{H}$. It will be therefore benefitial to start with some basic remarks on the general properties of the H-connection as well as of the coadjoint orbits of $\mathrm{H}$. After this we discuss inequivalent quantizations on the spheres $S^{n}$ for $n=2,3$ and 4 in detail ${ }^{7}$. We shall find that the cases $S^{2}, S^{4}$ have singular H-connections, which are identified with a Dirac monopole and a BPST (anti-)instanton, respectively. In fact, a singular and topologically non-trivial $\mathrm{H}$-connection is expected in a generic case where $\mathrm{G}$ is not (as a topological space) a direct product of $\mathrm{H}$ and $\mathrm{G} / \mathrm{H}$. The case $S^{3}$ is non-generic in this sense since $\mathrm{G}=\mathrm{SO}(4)$ is actually isomorphic to the direct product $\mathrm{SO}(4) \simeq \mathrm{SO}(3) \times S^{3}$ and hence the H-connection is tolopogically trivial. Nevertheless, the $S^{3}$ case is worth studying since it serves as a simple example where both the H-connection and the generalized spin emerge simultaneously. Furthermore, the generalized spin turns out to be just the usual $\operatorname{su}(2)$ spin, i.e., the conventional 'non-relativistic' spin degrees of freedom associated with the $\mathrm{SO}(3)$ frame rotations of the 'space'. The case $S^{4}$ is even more interesting in that the generalized spin consists of two $\mathrm{su}(2)$ spins, which couple to a BPST instanton and anti-instanton, chirally. As in the $S^{3}$ case, the chiral $\mathrm{su}(2)$ spins admit the interpretation that they are the two conventional 'relativistic' spins associated with the $\mathrm{SO}(4)$ frame rotations of the ‘space-time'.

\subsection{Some generalities on the H-connection}

The canonical H-connection has been studied earlier, e.g., in the context of the KaluzaKlein theories and in constructing topologically non-trivial Yang-Mills solutions [30]. For completeness we wish to recall here some of the basic properties of the H-connection which we shall need later. We begin by noting two salient features of the H-connection which follow directly from the definition (5.16). The first, which concisely charcterizes the Hconnection, is that its curvature $F$ is constant in the vielbein frame and given by the

7 The general $S^{n}$ is considered, though in less detail for the cases presented here, in [17] which is essentially based on the canonical group approach [8] (see also [18]). 
structure constants of the algebra $\mathfrak{g}$. To see this, observe first that the Maurer-Cartan equation for the left-invariant 1 -form $\sigma^{-1} d \sigma$,

$$
d\left(\sigma^{-1} d \sigma\right)+\left(\sigma^{-1} d \sigma\right) \wedge\left(\sigma^{-1} d \sigma\right)=0
$$

is equivalent to a pair of equations,

$$
d A+A \wedge A+\left.e \wedge e\right|_{\mathfrak{h}}=0 \quad \text { and } \quad d e+e \wedge A+A \wedge e+\left.e \wedge e\right|_{\mathfrak{r}}=0 .
$$

One then finds that the first equation in (6.2), which is identical to

$$
F=-\left.e \wedge e\right|_{\mathfrak{h}}
$$

implies that the curvature in the vielbein frame $F=\frac{1}{2} F_{a b}^{i} T_{i} e^{a} \wedge e^{b}$ has the components which are precisely the structure constants:

$$
F_{a b}^{i}=-f_{a b}^{i}
$$

Incidentally, we mention that the second equation in (6.2) can be written as

$$
D e=-\left.e \wedge e\right|_{\mathfrak{r}},
$$

where $D$ is the covariant derivative. This means that the vielbein is convariantly constant $D e=0$ if $\mathrm{G} / \mathrm{H}$ is a symmetric space. (It is worth noting that for any coset $\mathrm{G} / \mathrm{H}$ one can define a Riemannian spin connection 1-form satisfying the metricity and the torsionless conditions, leading to a Riemannian manifold with a constant curvature 2-form. In particular, for a symmetric $\mathrm{G} / \mathrm{H}$ the spin connection is essentially equivalent to the H-connection.)

The second, more important, feature is that the H-connection obeys the Yang-Mills equation on the coset space $\mathrm{G} / \mathrm{H}$, that is,

$$
D^{*} F=0
$$

where $D^{*}:=-* D *$ is the adjoint operator of $D$. We give a direct proof of this remarkable property in Appendix D for a semisimple, compact G (an alternative proof can be found, e.g., in [31]). In passing we wish to note that, for $\mathrm{G} / \mathrm{H}=\mathrm{SO}(2 m+1) / \mathrm{SO}(2 m) \simeq S^{2 m}$, it is known [30] that if the $\mathrm{H}$-connection is given in the spinor representation of $\mathrm{so}(2 m+1)$ then it can be decomposed into the two spinor representations of so $(2 m)$ such that one is 
self dual and the other anti-self dual (in the generalized sense). Thus, in this case, the fact that the $\mathrm{H}$-connection being a solution of the Yang-Mills equation on $\mathrm{G} / \mathrm{H}$ follows directly from the Bianchi identity $D F=0$.

Since the H-connection is necessarily singular when the prinipal bundle $\mathrm{G}$ is nontrivial, the construction of the H-connection provides - in principle - a method for obtaining topologically non-trivial solutions of the Yang-Mills equations on the coset G/H. (In fact, the foregoing argument already suggests that the H-connection would be a Dirac monopole potential for $\mathrm{G} / \mathrm{H}=\mathrm{SO}(3) / \mathrm{SO}(2) \simeq S^{2}$, or a BPST instanton for $\mathrm{G} / \mathrm{H}=\mathrm{SO}(5) / \mathrm{SO}(4) \simeq S^{4}$, which we shall confirm soon.) However, in practice, we need to make some technical remarks on the situation where such a topologically non-trivial solution actually occurs. For simplicity let us confine ourselves to the situation where $\mathrm{G} / \mathrm{H}$ is an even dimensional, symmetric space. In this situation one has $F=-e \wedge e$ (i.e., without projection to $\mathfrak{h}$ ) and hence the original H-connection given by some representation of $\mathfrak{g}$ must have a vanishing Chern number as a whole,

$$
\operatorname{tr}(\overbrace{F \wedge \cdots \wedge F}^{m \text { times }})=(-1)^{m} \operatorname{tr}(\overbrace{e \wedge \cdots \wedge e}^{2 m \text { times }})=0 .
$$

This, of course, does not mean that the H-connection must necessarily be topologically trivial when it is expressed in an irreducible representation of $\mathfrak{h}$.

To be more explicit, let $\left\{\pi^{\chi}\left(T_{i}\right)\right\}$ be the matrix form of a basis $\left\{T_{i}\right\}$ in $\mathfrak{h}$ in the irreducible representation $\chi$ of $\mathfrak{h}$. Then, given a curvature $F$ in some representation of $\mathfrak{g}$, we may decompose it into irreducible representations $\chi$ of $\mathfrak{h}$ as

$$
F=\sum_{\chi} \oplus F^{\chi}, \quad \text { where } \quad F^{\chi}:=\pi^{\chi}(F)=-\frac{1}{2} f_{a b}^{i} \pi^{\chi}\left(T_{i}\right) e^{a} \wedge e^{b}
$$

The Chern number for each $F^{\chi}$ is then proportional to

$$
\begin{aligned}
\operatorname{tr}(\overbrace{F^{\chi} \wedge \cdots \wedge F^{\chi}}^{m \text { times }})=\left(-\frac{1}{2}\right)^{m} & \varepsilon^{a_{1} b_{1} \ldots a_{m} b_{m}} f_{a_{1} b_{1}}^{i_{1}} \cdots f_{a_{m} b_{m}}^{i_{m}} \\
& \times \operatorname{tr}\left(\pi^{\chi}\left(T_{i_{1}}\right) \cdots \pi^{\chi}\left(T_{i_{m}}\right)\right) \frac{\Omega}{\sqrt{\operatorname{det} \eta_{a b}}},
\end{aligned}
$$

where $\Omega=\sqrt{\operatorname{det} \eta_{a b}} e^{1} \wedge \cdots \wedge e^{2 m}$ is the volume form of the coset space G/H. This decomposition may give us a non-trivial H-connection in some representation $\chi$ of $\mathfrak{h}$. But if, in particular, the coset space $\mathrm{G} / \mathrm{H}$ is four dimensional (i.e., $m=2$ ), and if $\mathfrak{h}$ is simple and compact, then for any $F^{\chi}$ the Chern number is zero. Indeed, for such $\mathfrak{h}$ every 
$\chi$ (apart from the trivial one) is faithful and has the quadratic trace proportional to the Killing form with the same sign. From (6.9) this means that the Chern numbers for all $F^{\chi}$ have the same sign, but since from (6.7) they must add up to zero when summed over the $\chi$, they must be all zero. If, however, $\mathfrak{h}$ is semisimple but not simple, then $F^{\chi}$ may have a non-vanishing Chern number because such $\mathfrak{h}$ admits non-faithful (but non-trivial) representations. Actually, this is the case we shall encounter later when we consider the quantization on $S^{4}$.

\subsection{Some generalities on the coadjoint orbits of $\mathrm{H}$}

The coadjoint orbits of a group have also been well studied; for instance, it is known that the orbits are Kähler manifolds, among which the minimal dimensional ones are completely classified for all classical groups, while the maximal dimensional ones — which arise for regular semisimple $K$ and are just $O_{K}=\mathrm{H} / \mathrm{T}$ with $\mathrm{T}$ being the maximal torus $\mathrm{T}$ of $\mathrm{H}$ - are flag manifolds (see, for example [7, 19, 25]). Although we do not need any of these special properties of the coadjoint orbits later, we shall need the following two. The first is quite simple: the variables $S_{i}=-\operatorname{Tr}\left(T_{i} h^{-1} K h\right)$ defined in (5.17) describe the generalized spin. This is based on the fact that they form the 'generalized spin algebra' in the reduced system, that is, their Dirac bracket (defined with respect to the second class constraints $\varphi_{k}=\left(\phi_{i}, \xi_{s}\right)$ considered in Sect. 5) is the algebra $\mathfrak{h}$ itself:

$$
\left\{S_{i}, S_{j}\right\}^{*}=f_{i j}^{k} S_{k}
$$

To prove this, note that $L_{O_{K}}$ would have been the only term in the total Lagrangian (5.15) if our reduction had been $\mathrm{H} / \mathrm{H}$ (rather than $\mathrm{G} / \mathrm{H}$ ) where the entire right-currents $R_{i}$ are constrained to be $K_{i}$. Obviously, in this reduction the left-currents $L_{i}$ in (4.3), which reduce to $S_{i}$ on the constrained surface, commute with the constraints under Poisson bracket and thus are gauge invariant. It then follows that the Dirac bracket among $L_{i}$ is equivalent to the Poisson bracket in the reduced system (i.e., on the second class constrained surface) and hence we get $(6.10)^{8}$. Upon quantization, the Dirac bracket (6.10) is replaced by the quantum commutator,

$$
\left[\widehat{S}_{i}, \widehat{S}_{j}\right]=i f_{i j}^{k} \widehat{S}_{k}
$$

8 In other words, just as we saw for the spin example in Sect. 3, the system of the coadjoint orbit $O_{K}$ of $\mathrm{H}$ can be obtained simply by the reduction $\mathrm{H} \rightarrow \mathrm{H} / \mathrm{H}$ in our generalized Dirac approach. The same technique can be applied even to loop groups LG, where one finds, e.g., the model of non-abelian chiral bosons as a coadjoint orbit LG/G [32]. 
In view of the quantum theory discussed in Sect. 4, the Hilbert space on which the generalized spin is represented is given by the highest weight representation $\chi$ of $\mathrm{H}$ with $\chi\left(H_{\alpha_{r}}\right)=K_{r}$.

The second property we wish to note is slightly more involved; it is on the 'space-time' nature of the generalized spin given by the coadjoint orbit $O_{K}$. Let us observe that the change of section (5.19) induces, in addition to the gauge transformation (5.20) for the $\mathrm{H}$-connection, the following transformation for the vielbein,

$$
e=e^{a} T_{a} \longrightarrow \tilde{h}^{-1} e \tilde{h}=e^{a} M_{a}^{b}(\tilde{h}) T_{b}, \quad \text { with } \quad M_{a}^{b}(\tilde{h}):=\eta^{b c} \operatorname{Tr}\left(\tilde{h}^{-1} T_{a} \tilde{h} T_{c}\right)
$$

where $\left\{T_{a}\right\}$ is a basis in $\mathfrak{r}$, which specifies the (vielbein) frame in the tangent space defined at each point on the coset space $\mathrm{G} / \mathrm{H}$. Since (6.12) leaves the metric $g_{\alpha \beta}$ invariant, it is an $\mathrm{SO}(n)(n=\operatorname{dim}(\mathrm{G} / \mathrm{H}))$ rotation of the vielbein frame. To put it differently, on account of the reductive decomposition (A.6) the complement $\mathfrak{r}$ automatically furnishes a linear space for a representation of the group $\mathrm{H}$ by the adjoint action (6.12), producing the $\mathrm{SO}(n)$ frame rotation. In fact, we shall see that for $S^{3}$ the representation in the vielbein frame is given by the adjoint of $\mathrm{SU}(2)$, that is, the defining representation of $\mathrm{SO}(3)$, and hence provides the totality of the frame rotation. A similar situation occurs for $S^{4}$ where again the representation in the vielbein frame by the adjoint action (6.12) provides the totality of the frame rotation $\mathrm{SO}(4)$. Thus, combined with the response (2.18) under the change of section in the sector $\chi$, this observation enables us to determine the representation of the generalized spin under the frame rotation in those cases.

\subsection{Quantizing on $S^{n} \simeq \mathrm{SO}(n+1) / \mathrm{SO}(n)$}

We are now in a position to elaborate the physical consequences of inequivalent quantizations by the examples of a particle moving on the sphere $S^{n}$, realized as the coset $\mathrm{SO}(n+1) / \mathrm{SO}(n)$, for $n=2,3$ and 4 . Our aim is to examine the role played by the generalized spin, as well as to find explicitly what the H-connections are. We begin with the simplest case $S^{2} \simeq \mathrm{SO}(3) / \mathrm{SO}(2)$.

\section{i) $S^{2}-$ Dirac monopole:}

Let us choose for our basis $T_{m}=\frac{\sigma_{m}}{2 i}$ for which $\operatorname{tr}\left(T_{m} T_{n}\right)=-\frac{1}{2} \delta_{m n}$, and take the orthogonal decomposition of $\mathfrak{g}$ as

$$
\mathfrak{g}=\mathfrak{h} \oplus \mathfrak{r}=\operatorname{span}\left\{T_{3}\right\} \oplus \operatorname{span}\left\{T_{1}, T_{2}\right\}
$$


Although the H-connection does emerge in this case, no spin degrees of freedom appear (since the coadjoint orbits of $\mathrm{SO}(2)$ are points). Thus the total Lagrangian (5.15) reduces to

$$
L_{\mathrm{tot}}=\frac{1}{2} g_{\alpha \beta}(q) \dot{q}^{\alpha} \dot{q}^{\beta}-\operatorname{Tr}\left(K A_{\alpha}(q)\right) \dot{q}^{\alpha},
$$

where $K=j T_{3}$. If we set the radius of $S^{2}$ to unity, then from Appendix C we find $c_{\chi_{\mathrm{sp}}}=\frac{1}{2}$, which means that our metric in the vielbein frame is $\eta_{a b}=\delta_{a b}$. The curvature $F$ of the $\mathrm{H}$-connection is just

$$
F=-\frac{1}{2}\left[T_{a}, T_{b}\right] e^{a} \wedge e^{b}=-T_{3} \Omega,
$$

with $\Omega=e^{1} \wedge e^{2}$ being the volume form of the unit sphere $S^{2}$, which implies that the $\mathrm{H}$-connection is a Dirac monopole potential on $S^{2}$ as expected.

It is reassuring to see that the Dirac condition for the monopole charge follows directly from the quantization condition of the parameter in $K$. Indeed, since the effective abelian H-connection coupled to the particle is $\bar{A}:=-\operatorname{Tr}(K A)$ and hence the effective curvature $\bar{F}=-j \operatorname{Tr}\left(T_{3} F\right)=j \Omega$, the monopole charge $g-$ given by $\left(-\frac{1}{2}\right)$ times the first Chern number $C_{1}$ - is found to be

$$
g=-\frac{1}{2} C_{1}[\bar{F}]=\frac{1}{4 \pi} \int_{S^{2}} \bar{F}=\frac{j}{4 \pi} \int_{S^{2}} \Omega=j .
$$

Then by the parameter quantization $j \in \mathbb{Z}$ (see Sect.3) we recover the Dirac condition for the monopole charge. (Had we started with $S^{2} \simeq \mathrm{SU}(2) / \mathrm{U}(1)$, then on account of $2 j \in \mathbb{Z}$ we would have seen $g$ to be a multiple of $\frac{1}{2}$ - the standard result for the monopole charge in that setting.)

ii) $S^{3}$ - emergence of spin:

The case $S^{3}$ is doubly exceptional; first, it is a group manifold $S^{3} \simeq \mathrm{SU}(2)$ by itself, and thus one can quantize without regarding $S^{3}$ as a coset, yielding a unique quantum theory where neither the H-connection nor generalized spin occur. Second, even if one regards $S^{3}$ as the coset $\mathrm{SO}(4) / \mathrm{SO}(3)$, on account of the direct product structure ${ }^{9}$ of the space $\mathrm{SO}(4) \simeq \mathrm{SO}(3) \times S^{3}$ one finds that the $\mathrm{H}$-connection is topologically trivial (although its curvature is non-vanishing). Nonetheless, this example is very interesting not only in that it provides one of the simplest coset spaces where we find both of the effects - the

9 Another $\mathrm{SO}(\mathrm{n})$ which admits a similar direct product is $\mathrm{SO}(8) \simeq \mathrm{SO}(7) \times S^{7}$. 
H-connection and the generalized spin — but also in that the generalized spin can be regarded as the conventional $\mathrm{su}(2)$ spin attached to the particle.

To see this, we shall work in the defining representation $\chi_{\text {def }}$ of $\mathrm{SO}(4)$ with the basis $\left\{T_{i j}\right\}$ given in (C.5). Using $T_{i}:=-\frac{1}{2} \varepsilon_{i j k} T_{j k}$ and $T_{a}:=T_{a 4}$, we take the decomposition,

$$
\mathfrak{g}=\mathfrak{h} \oplus \mathfrak{r}=\operatorname{span}\left\{T_{i}\right\} \oplus \operatorname{span}\left\{T_{a}\right\}, \quad i, a=1,2,3
$$

The basis $\left\{T_{i}\right\}$ forms an su(2) algebra $\left[T_{i}, T_{j}\right]=\varepsilon_{i j k} T_{k}$, with respect which the basis in $\mathfrak{r}$ becomes a (spin 1) vector,

$$
\left[T_{i}, T_{a}\right]=\varepsilon_{i a b} T_{b}
$$

Note that from the argument given earlier the variables $S_{i}$ form an $\mathrm{su}(2)$ algebra under the Dirac bracket,

$$
\left\{S_{i}, S_{j}\right\}^{*}=\varepsilon_{i j k} S_{k}
$$

This is also consistent with the fact that the Lagrangian $L_{O_{K}}$ for the coadjoint orbit becomes the usual spin Lagrangian $L_{\text {spin }}=-j \operatorname{Tr} T_{3}\left(h^{-1} \dot{h}\right)$ discussed in Sect. 3. Furthermore, since the basis $\left\{T_{a}\right\}$ forms a vector (6.18), the adjoint action (6.12) yields the usual adjoint representation of the group $\mathrm{SU}(2)$, i.e., the defining representation of $\mathrm{SO}(3)$. This implies that any $\mathrm{SO}(3)$ rotation of the vielbein frame is realized by the adjoint action (6.12), under which the spin $S_{i}$ furnishes the representation of total spin $j$ - the conventional picture of 'non-relativistic' spin responding under the $\mathrm{SO}(3)$ rotations in 'space'. Thus we see that quantizing on $S^{3}$ provides another example (besides Mackey's account of spin by quantizing on $\mathbb{R}^{3}$ ) in which the particle acquires spin due to the inequivalent quantizations.

On the other hand, the H-connection is regular everwhere on $S^{3}$ since there exists a globally well defined section. Thus this $S^{3}$ case serves as a concrete example where the parameter quantization of $K$ (or the quantization of the coupling constants) is not linked to the singular nature of the H-connection. This is in contrast to the generic situation where the two - the singularity of the H-connection and the parameter quantization are closely linked (e.g., the quantization of a Dirac monopole charge is often derived by requiring the insensitivity of the path-integral to the location of the singularity).

Finally, we mention an ambiguity in the reductive decomposition: in general the reductive decomposition is far from unique, and the properties of the H-connection and the generalized spin depend on the decomposition. For example, in the present case we may take, instead of (6.17), the decomposition of so(4) into two commuting su(2) subalgebras 
as $\mathrm{so}(4)=\mathrm{su}(2) \oplus \mathrm{su}(2)$. Then, using this, we will find that the H-connection vanishes identically on $S^{3}$; only the spin degrees of freedom appear but decoupled from the free particle moving on $S^{3}$. Moreover, the relation in the group representation of $\mathrm{H}$ between the frame rotation and the spin will be lost; the frame rotation becomes completely independent from the $\mathrm{SU}(2)$ group action of the spin. Thus this new reductive decomposition does not provide the conventional spin in this respect.

iii) $S^{4}$ - BPST instanton/anti-instanton and two chiral spins:

The case $S^{4}$ allows for a more intriguing physical interpretation of the consequences of the inequivalent quantizations; we shall see that the H-connection consists of a BPST instanton and anti-instanton, and that the generalized spin splits into two $\mathrm{su}(2)$ spins coupled chirally to the instanton and anti-instanton, respectively. To show this, we use the spinor representation of so(5), and choose for our basis $\left\{T_{m}\right\}=\left\{T_{a}, T_{i}\right\}$ with

$$
T_{a}= \begin{cases}\frac{1}{2 i}\left(\begin{array}{r}
\sigma_{a} \\
\sigma_{a}
\end{array}\right), & \text { for } a=1,2,3, \\
\frac{1}{2}\left(\begin{array}{r}
-1 \\
1
\end{array}\right), & \text { for } a=4\end{cases}
$$

and

$$
T_{i}= \begin{cases}\frac{1}{2 i}\left(\begin{array}{cc}
\sigma_{i} & \\
& \sigma_{i}
\end{array}\right), & \text { for } i=1,2,3, \\
\frac{1}{2 i}\left(\begin{array}{cc}
\sigma_{i-3} & \\
& -\sigma_{i-3}
\end{array}\right), & \text { for } i=4,5,6,\end{cases}
$$

for which $\operatorname{tr}\left(T_{m} T_{n}\right)=-\delta_{m n}$. The decomposition is taken to be

$$
\mathfrak{g}=\mathfrak{h} \oplus \mathfrak{r}=\operatorname{span}\left\{T_{i}\right\} \oplus \operatorname{span}\left\{T_{a}\right\}
$$

Adopting the unit radius $S^{4}$, we find $c_{\chi_{\mathrm{sp}}}=1$ from Appendix $\mathrm{C}$ and hence the metric in the vielbein frame is $\eta_{a b}=\delta_{a b}$.

Recall that for any $m$ the spinor representation of so $(2 m+1)$, when restricted to so $(2 m)$, is decomposed into the two spinor representations of so $(2 m)$. In the present case $2 m=4$ the two so(4) spinor representations given in (6.21) are reducible, which of course is due to the direct sum structure $\mathrm{so}(4)=\mathrm{su}(2) \oplus \mathrm{su}(2)$. Thus it is already clear that we have two $\mathrm{su}(2)$-valued variables for our generalized spin. In order to treat the two $\mathrm{su}(2)$ separately, which we label by $\mathrm{su}(2)^{+}$and $\mathrm{su}(2)^{-}$, it is convenient to introduce the chiral basis

$$
T_{i}^{+}=\frac{1}{2}\left(T_{i}+T_{i+3}\right)=\frac{1}{2 i}\left(\begin{array}{cc}
\sigma_{i} & \\
& 0
\end{array}\right), \quad T_{i}^{-}=\frac{1}{2}\left(T_{i}-T_{i+3}\right)=\frac{1}{2 i}\left(\begin{array}{cc}
0 & \\
& \sigma_{i}
\end{array}\right),
$$


for $i=1,2,3$, and thereby write $h=h^{+} h^{-}$, where $h^{ \pm}$belong to the exponential groups generated by the chiral su(2) ${ }^{ \pm}$in the basis (6.23). Setting $K=j^{+} T_{3}^{+}+j^{-} T_{3}^{-}$, we find that the Lagrangian for the coadjoint orbit of $\mathrm{SO}(4)$ consists of those for the coadjoint orbits of the two $\mathrm{su}(2)$, i.e., for the two conventional spins,

$$
L_{O_{K}}=L_{\mathrm{spin}}^{+}+L_{\mathrm{spin}}^{-}=-j^{+} \operatorname{Tr} T_{3}^{+}\left(\left(h^{+}\right)^{-1} \dot{h}^{+}\right)-j^{-} \operatorname{Tr} T_{3}^{-}\left(\left(h^{-}\right)^{-1} \dot{h}^{-}\right) .
$$

Moreover, from (6.10) we have the variables $S_{i}^{ \pm}=-j^{ \pm} \operatorname{Tr}\left(T_{i}^{ \pm}\left(h^{ \pm}\right)^{-1} T_{3}^{ \pm} h^{ \pm}\right)$forming two commuting su(2) algebras under the Dirac bracket,

$$
\left\{S_{i}^{+}, S_{j}^{+}\right\}^{*}=\varepsilon_{i j k} S_{k}^{+}, \quad\left\{S_{i}^{-}, S_{j}^{-}\right\}^{*}=\varepsilon_{i j k} S_{k}^{-}, \quad\left\{S_{i}^{+}, S_{j}^{-}\right\}^{*}=0 .
$$

From the relations,

$$
\left[T_{i}^{ \pm}, T_{a}\right]=\frac{1}{2} \varepsilon_{i a b} T_{b} \pm \frac{1}{2} \delta_{i a} T_{4}, \quad\left[T_{i}^{ \pm}, T_{4}\right]=\mp \frac{1}{2} \delta_{i a} T_{a},
$$

it is also easy to see that the basis $\left\{T_{ \pm \pm}:=T_{1} \mp i T_{2}, T_{ \pm \mp}:=T_{3} \mp i T_{4}\right\}$ in the space $\mathfrak{r}$ forms a tensor product representation $2^{+} \otimes 2^{-}$of spin $\frac{1}{2}$ with respect to each chiral $\operatorname{su}(2)^{ \pm}$. Accordingly, the adjoint action (6.12) of $\mathrm{H}=\mathrm{SO}(4)$ amounts to the product $\mathrm{SU}(2) \times \mathrm{SU}(2)$ transformation in the vielbein frame. However, taking into account the fact that the spinor representations of $\mathrm{SO}(4)$ are 'double-valued' as a group representation, we conclude that the 'true group' described by this adjoint action is $(\mathrm{SU}(2) \times \mathrm{SU}(2)) / \mathbb{Z}_{2}$, which is the totality of the frame rotation $\mathrm{SO}(4)$. (In other words, the representation of $\mathrm{H}=\mathrm{SO}(4)$ in the vielbein frame is faithful and 'single-valued', and thus the group action of $\mathrm{H}$ has a one to one correspondence to the $\mathrm{SO}(4)$ frame rotation.) This is exactly the same situation encountered in the Minkowski space version of the frame rotation, where the Lorentz frame rotation (that is, the action of the proper, orthochronous Lorents group) is realized by the group action of $\mathrm{SL}(2, \mathbb{C})$ consisting of two chiral $\mathrm{SU}(2)$ actions. Thus we have seen that, on $S^{4}$, we recover the conventional, two su(2) chiral 'relativistic' spins from the inequivalent quantizations.

Turning to the H-connection, we observe that the su(2)-valued H-connections $A^{ \pm}$, defined by the decomposition $A=A^{+}+A^{-}$in terms of the chiral basis (6.23), couple to the chiral su(2) spins chirally,

$$
L_{\mathrm{int}}=\operatorname{Tr}\left(S^{+} A_{\alpha}^{+}\right) \dot{q}^{\alpha}+\operatorname{Tr}\left(S^{-} A_{\alpha}^{-}\right) \dot{q}^{\alpha} .
$$


We now show that these chiral H-connections $A^{ \pm}$are nothing but a BPST instanton and anti-instanton, respectively. We do this by computing explicitly the Chern number of each of the $\mathrm{su}(2)$-valued $\mathrm{H}$-connection $A^{ \pm}$; from (6.9) we get

$$
F^{ \pm} \wedge F^{ \pm}=\frac{1}{4} \varepsilon^{a b c d} f_{a b}^{i} f_{c d}^{j} T_{i}^{ \pm} T_{j}^{ \pm} \Omega
$$

where $F^{ \pm}$are the curvatures corresponding to $A^{ \pm}$and $\Omega=e^{1} \wedge e^{2} \wedge e^{3} \wedge e^{4}$ is the volume form on $S^{4}$ with unit radius, $\int_{S^{4}} \Omega=\frac{8 \pi^{2}}{3}$. The structure constants appearing in (6.28) can be read off from the commutation relations,

$$
\left[T_{a}, T_{b}\right]=\varepsilon_{a b i} T_{i} \quad \text { and } \quad\left[T_{a}, T_{4}\right]=\delta_{a, i-3} T_{i}
$$

for $a, b=1,2,3$. Then the instanton number for $F^{ \pm}$- given by $(-1)$ times the second Chern number $C_{2}$ - is evaluated to be

$$
n^{ \pm}=-C_{2}\left[F^{ \pm}\right]=-\frac{1}{8 \pi^{2}} \int_{S^{4}} \operatorname{tr}\left(F^{ \pm} \wedge F^{ \pm}\right)=-\frac{1}{8 \pi^{2}} \cdot \frac{8 \pi^{2}}{3} \cdot(\mp 3)= \pm 1,
$$

which shows that $A^{ \pm}$is indeed a BPST instanton (anti-instanton). One can also confirm that $F^{ \pm}$satisfies the self dual (anti-self dual) equation:

$$
* F^{+}=F^{+}, \quad * F^{-}=-F^{-} .
$$

We finish this section by remarking on a techincal point underlying the topological property of the H-connection. We have argued in Sect.6.1 that for a four dimensional symmetric space $\mathrm{G} / \mathrm{H}$ the $\mathrm{H}$-connection must necessarily be topologically trivial if $\mathfrak{h}$ is a simple algebra. This however does not apply to the above case; so(4) is semisimple but not simple. In fact, the two $\mathrm{su}(2)$ representations given in the chiral basis (6.23) are not faithful representations of $\mathrm{so}(4)$, which allows the $\mathrm{H}$-connection to escape from the argument given there, although the total sum of the two Chern numbers is zero as required in (6.7). 


\section{Discussion}

In this paper we have seen that Dirac's formulation of constrained quantization has to be generalized in order to recover the known quantizations on the coset space $\mathrm{G} / \mathrm{H}$ by reducing from the naive quantization on $\mathrm{G}$. In this generalization the classical first class constraints $R_{i}=0$ become, in the quantum theory, a mixed set of constraints $R_{i}=K_{i}$, where the constants $K_{i}$ label the irreducible representations of $\mathrm{H}$. We have developed a path-integral account of this within which the quantization of the constants $K_{i}$ follow from the residual $\mathrm{S}_{K}$-invariance generated by the first class subset of the constaints. This path-integral formulation clearly shows how the different reductions are associated with additional, compact degrees of freedom which we identify with generalized spin, and how the effective dynamics is described in terms of a minimal coupling to the H-connection.

The generalization we have presented here is not unique. Indeed it is also possible to recover the quantizations on $\mathrm{G} / \mathrm{H}$, while still keeping with the original constraint, by modifying the first step in the constrained quantization process [33]; that is, to keep with one reduction but to allow for many quantizations on $\mathrm{G}$ by identifying it with the coset

space $(\mathrm{G} \times \mathrm{H}) / \widetilde{\mathrm{H}}$, where $\widetilde{\mathrm{H}}$ is the diagonal subgroup of $\mathrm{G} \times \mathrm{H}$. Then, appealing to Mackey's analysis of such a coset space, there will now be many inequivalent quantizations on $G$ labelled by the irreducible representations of $\widetilde{\mathrm{H}} \simeq \mathrm{H}$. The reduction to the quantizations on $\mathrm{G} / \mathrm{H}$ can now be accomplished by applying the standard Dirac approach to these many quantizations on G. Although this procedure has ended up with the correct result, we do not feel that it is a satisfactory constrained method since it still involves the use of vectorvalued wave functions on $\mathrm{G}$, hence obstructing a continuous path-integral formulation. Besides, the reliance on a non-trivial quantization on the extended configuration space cannot be extended in any obvious way to field theory (a fuller account of these points is presented in [23]).

First class constraints generate symmetries. In these quantum mechanical examples the symmetries are local in time, and are thus the natural model for the gauge symmeries (local in space and time) found in Yang-Mills theories. The most startling aspect of our analysis is that we have seen that these classical symmetries do not, and should not, always go through to the quantum theory. Thus, contrary to our normal expectation, anomalous behaviour is the norm when quantizing gauge theories! Of course, in Yang-Mills theory coupled to chiral fermions, the naive quantization is being used: it then being the matter fields that cause the anomalous structure. Indeed, the chiral anomaly is probing the 
topology of the Yang-Mills configuration space, and is, in fact, reflecting the existence of non-contractable two-spheres in the reduced configuration space [34]. However, due to the very existence of these topological structures, we expect there to be non-trivial quantizations possible such that the resulting anomalous structure will be the sum of that comming from the chiral fermions and that comming from the particular quantum sector one is working in. That such topological structures lead to inequivalent quantizations in Yang-Mills theory follows quite naturally from our constrained formulation and will be presented elsewhere [11]. The resulting functional connections, generalizing the $\mathrm{H}$ connection encountered in this paper, will be seen to generalize the results of [28].

The situation here is, we feel, analogous to that found when discussing the AharonovBohm effect [35]. In this situation there is an inaccessible region in the plane through which a flux is passing, resulting in the charged particles picking up a phase when they pass around that region. This phase is apparently well defined and corresponds to the flux through the system. However, if the region through which the flux is passing is really inaccessible to the particles, then the configuration space is topologically a circle and there will be inequivalent quantizations labelled by a phase ${ }^{10}$. Thus the observed phase is really the sum of two terms, one representing the actual flux put into the system by hand, the other determined by the particular quantum sector one is in. The intriguing point in the chiral gauge theory example is then to determine whether it is possible to quantize such that the resulting amomalies coming from the two sources cancel each other.

Extending this argument, we note that there appears to be some connection between the inequivalent quantizations described in this paper and the geometric phase that has become popular in physics. Indeed, the emergence of a monopole when quantizing on a two-sphere is reminiscent of the occurence of a monopole when an adiabatic transport is performend in a parameter space which is a two-sphere [36]. A non-abelian generalization of Berry's phase is possible [37] such that the parameter space is an $n$-sphere, and the associated H-connection (in this case an $\mathrm{SO}(\mathrm{n})$-connection) emerges. (For a more general framework based on the generalized coherent state path-integral, see [38].) In these discussions the naive quantization is always being used, so we would again expect that the resulting observed phases should also have a decomposition into the naive term and

10 The phase here is characterizing the irreducible representations of the group of integers $\mathbb{Z}$; that is we are identifying $S^{1}=\mathbb{R} / \mathbb{Z}$ and applying Mackey to this coset space. A similar situation arises in gauge theories with the emergence of the $\theta$-vacua [2]. 
a part labeling the quantum sector of the theory. Such a point of view will be developed elsewhere.

In conclusion, we have presented here a constrained description of how inequivalent quantizations arise. What is missing is a constrained account of why they arise (a possible account is given in [23]). However, we hope that the methods presented here will, at least, help to make the important ideas of Mackey seem more accessible, and indeed more relevant, to the general physics community.

Acknowledgements: We both wish to thank John Lewis and Lochlainn O'Raifeartaigh for their support in this work, and I.T. wishes to thank László Fehér for discussions. 


\section{Appendix A. Conventions}

In this appendix we shall provide our conventions along with some basic facts of Lie groups/algebras $[21,26]$ used in the text.

Innerproduct. Let $\mathrm{G}$ be a semisimple group and $\mathfrak{g}$ its Lie algebra. Using some irreducible representation $\chi$ of the algebra $\mathfrak{g}$, one can define in $\mathfrak{g}$ an innerproduct $(\cdot, \cdot)$ by the formula

$$
(A, B)=\operatorname{Tr}(A B), \quad \text { where } \quad \operatorname{Tr}(A B):=-\frac{1}{c_{\chi}} \operatorname{tr}\left(\pi^{\chi}(A) \pi^{\chi}(B)\right),
$$

where $\pi^{\chi}(A), \pi^{\chi}(B)$ are the matrices in the representation $\chi$ which correspond to $A$, $B \in \mathfrak{g}$. (When it is obvious, we often omit $\pi^{\chi}$ for brevity.) For a simple group $\mathrm{G}$ the (positive) constant $c_{\chi}$ is related to the index of the representation, which is inserted to make the innerproduct representation-independent. Note however that the constant $c_{\chi}$ can be rescaled freely; only the relative normalizations among representations are fixed.

Highest weight representation. In $\mathfrak{g}$, or in the complex extension $\mathfrak{g}_{\mathrm{c}}$ of $\mathfrak{g}$, one can choose (with respect to some Cartan subalgebra) the Chevalley basis $\left\{H_{\alpha}, E_{ \pm \varphi}\right\}$ where $\alpha$ are simple roots and $\varphi$ are positive roots. The basis has the relations

$$
\left[E_{\alpha}, E_{-\alpha}\right]=H_{\alpha}, \quad\left[H_{\alpha}, E_{\beta}\right]=K_{\beta \alpha} E_{\beta},
$$

for simple roots $\alpha, \beta$, and $K_{\beta \alpha}=\beta\left(H_{\alpha}\right)=\frac{2 \beta \cdot \alpha}{|\alpha|^{2}}$ is the Cartan matrix. To every dominant weight $\chi$ there exists an irreducible representation — highest weight representation - of $\mathfrak{g}$ where the Cartan elements $H_{\alpha}$ are diagonal; in particular, on the states $|\chi, \mu\rangle$ specified by the weights $\mu$ connected to the the dominant weight $\chi$ (identified as the highest weight in the representation) their eigenvalues are all integer:

$$
H_{\alpha}|\chi, \mu\rangle=\mu\left(H_{\alpha}\right)|\chi, \mu\rangle, \quad \text { with } \quad \mu\left(H_{\alpha}\right)=\frac{2 \mu \cdot \alpha}{|\alpha|^{2}} \in \mathbb{Z} .
$$

On account of this, we can use the dominant weight $\chi$ (or the set of integers $\chi\left(H_{\alpha_{r}}\right)$ for $r=1, \ldots$, rank G) to label the irreducible representation ${ }^{11}$. The integral property (A.3) of $H_{\alpha}$ leads to the periodicity in the exponential mapping defined in the universal covering group $\widetilde{\mathrm{G}}$ of $\mathrm{G}$ (i.e., the simply connected group which shares the same algebra $\mathfrak{g}$ ),

$$
e^{2 n \pi i H_{\alpha}}=1, \quad \text { for } n \in \mathbb{Z} .
$$

11 In the text we consider highest weight representations $\chi$ of a subalgebra $\mathfrak{h}$ of $\mathfrak{g}$, or of its group $\mathrm{H}$ for which we use the same notation. 
For a non-simply connected group G the periodicity is different from (A.4); it is mulitplied by a factor determined by the discrete normal subgroup $\mathrm{N}$ of $\widetilde{\mathrm{G}}$ for which $\mathrm{G} \simeq \widetilde{\mathrm{G}} / \mathrm{N}$. For instance, for $\operatorname{Spin}(n)=\widetilde{\mathrm{SO}}(n)$ we have $n \in \mathbb{Z}$ but for $\mathrm{SO}(n) \simeq \operatorname{Spin}(n) / \mathbb{Z}_{2}$ we find $2 n \in \mathbb{Z}$ $(\operatorname{Spin}(3)=\mathrm{SU}(2))$.

Reductive decomposition. Given a closed subgroup $\mathrm{H}$ of $\mathrm{G}$ with its Lie algebra $\mathfrak{h}$, we take an orthogonal decomposition of $\mathfrak{g}$,

$$
\mathfrak{g}=\mathfrak{h} \oplus \mathfrak{r},
$$

where $\mathfrak{r}=\mathfrak{h}^{\perp}$ is the orthogonal complement of $\mathfrak{h}$ to $\mathfrak{g}$ with respect to the innerproduct; $(\mathfrak{h}, \mathfrak{r})=0$. This is, in fact, a reductive decomposition, i.e.,

$$
[\mathfrak{h}, \mathfrak{r}] \subset \mathfrak{r}
$$

since $[\mathfrak{h}, \mathfrak{h}] \subset \mathfrak{h}$ and the orthogonality imply $0=([\mathfrak{h}, \mathfrak{h}], \mathfrak{r})=(\mathfrak{h},[\mathfrak{h}, \mathfrak{r}])$ and hence the relation (A.6). If, in addition, we have

$$
[\mathfrak{r}, \mathfrak{r}] \subset \mathfrak{h},
$$

then the coset space $\mathrm{G} / \mathrm{H}$, given by the left cosets $\{g \mathrm{H} \mid g \in \mathrm{G}\}$, is a symmetric space.

We shall denote bases of the spaces by

$$
\begin{aligned}
\mathfrak{g} & =\operatorname{span}\left\{T_{m}\right\}, & m & =1, \ldots, \operatorname{dim} \mathrm{G}, \\
\mathfrak{h} & =\operatorname{span}\left\{T_{i}\right\}, & i & =1, \ldots, \operatorname{dim} \mathrm{H}, \\
\mathfrak{r} & =\operatorname{span}\left\{T_{a}\right\}, & a & =1, \ldots, \operatorname{dim}(\mathrm{G} / \mathrm{H}) .
\end{aligned}
$$

As usual, any $A \in \mathfrak{g}$ can be expanded as $A=A^{m} T_{m}$ and one can raise or lower the indices by using the (flat) metric $\eta_{m n}:=\left(T_{m}, T_{n}\right)$ and its inverse $\eta^{m n}$ with $\eta^{m l} \eta_{l n}=\delta_{n}^{m}$. We use the structure constants $f_{m n}^{l}$ defined by $\left[T_{m}, T_{n}\right]=f_{m n}^{l} T_{l}$; in more detail,

$$
\left[T_{i}, T_{j}\right]=f_{i j}^{k} T_{k}, \quad\left[T_{i}, T_{a}\right]=f_{i a}^{b} T_{b}, \quad\left[T_{a}, T_{b}\right]=f_{a b}^{c} T_{c}+f_{a b}^{i} T_{i} .
$$

If $\mathrm{G} / \mathrm{H}$ is a symmetric space we have $f_{a b}^{c}=0$. If, on the other hand, $\mathrm{G}$ is compact, then the Killing form, defined by the matrix trace of the adjoint representation, is negative definite and hence we can find a basis where the metric takes the form $\eta_{m n}=\delta_{m n}$. Using this basis, we can ignore the differences between upper and lower indices, and the structure constants are totally antisymmetric in the three indices. In this paper we consider only semisimple, compact groups for $\mathrm{H}$, and the coset $\mathrm{G} / \mathrm{H}=\mathrm{SO}(n+1) / \mathrm{SO}(n)$, which is our main concern in Sect. 6, is a symmetric space. 


\section{Appendix B. Free particle on the group manifold G}

In this appendix we shall provide a Hamiltonian description of a free particle moving on the group manifold $\mathrm{G}$.

In general, a Hamiltonian system is defined by the triple $(M,\{\cdot, \cdot\}, H)$ where $M$ is the phase space, $\{\cdot, \cdot\}$ the Poisson bracket and $H$ the Hamiltonian. For our purpose we take for $M$ the cotangent bundle of the group $\mathrm{G}$,

$$
M=T^{*} \mathrm{G}=\mathrm{G} \times \mathfrak{g}^{*} \simeq \mathrm{G} \times \mathfrak{g}=\{(g, R) \mid g \in \mathrm{G}, R \in \mathfrak{g}\}
$$

The Poisson bracket can be given from the symplectic 2-form

$$
\omega=-d \operatorname{Tr} R\left(g^{-1} d g\right)=-d\left\{R_{m}\left(g^{-1} d g\right)^{m}\right\}
$$

and our Hamiltonian is

$$
H=\frac{1}{2} \operatorname{Tr} R^{2}=\frac{1}{2} \eta^{m n} R_{m} R_{n}
$$

In order to derive the Poisson bracket from the symplectic 2-form, we first recall that to every function $f=f(g, R)$ on $M$ there exists a corresponding Hamiltonian vector field $X_{f}$ satisfying

$$
i_{X_{f}} \omega:=\omega\left(X_{f}, \cdot\right)=-d f,
$$

where $i_{X_{f}}$ is the contraction with the vector field $X_{f}$. By using the left-invariant 1-forms $\left(g^{-1} d g\right)^{m}$ and their duals $Y_{m}$, the left-invariant vector fields for which $\left\langle\left(g^{-1} d g\right)^{m}, Y_{n}\right\rangle=$ $\delta_{n}^{m}$, one has

$$
d f=Y_{m} f \cdot\left(g^{-1} d g\right)^{m}+\frac{\partial f}{\partial R_{m}} d R_{m},
$$

and hence from (B.2) and (B.4) one finds

$$
X_{f}=-\frac{\partial f}{\partial R_{m}} Y_{m}+\left(Y_{m} f+f_{m n}^{l} \frac{\partial f}{\partial R_{n}} R_{l}\right) \frac{\partial}{\partial R_{m}} .
$$

Then the Poisson bracket between the two functions $f, h$ on $M$ is given by

$$
\{f, h\}:=\omega\left(X_{h}, X_{f}\right)=\frac{\partial f}{\partial R_{m}} Y_{m} h-\frac{\partial h}{\partial R_{m}} Y_{m} f+f_{m n}^{l} \frac{\partial f}{\partial R_{m}} \frac{\partial h}{\partial R_{n}} R_{l} .
$$

In particular, we have the following fundamental Poisson bracket,

$$
\left\{R_{m}, R_{n}\right\}=f_{m n}^{l} R_{l}, \quad\left\{R_{m}, g\right\}=Y_{m} g
$$


Under the use of some representation $\chi$, the left-invariant vector fields admit the explicit form $Y_{m}=\left(T_{m}\right)_{i j} g_{k i} \frac{\partial}{\partial g_{k j}}$. Using this, one finds that the second relation in (B.8) becomes $\left\{R_{m}, g_{i j}\right\}=\left(g T_{m}\right)_{i j}$, which confirms that the 'right-currents' $R_{m}$, or equivalently the left-invariant vector fields $Y_{m}$, are the generators of the right translation. It is also easy to see that the 'left-currents' $L_{m}$ defined by $L=-g R g^{-1}$ satisfy

$$
\left\{L_{m}, L_{n}\right\}=f_{m n}^{l} L_{l}, \quad\left\{L_{m}, g\right\}=-Z_{m} g
$$

where $Z_{m}$ are the right-invariant vector fields dual to the right-invariant 1-forms $\left(d g g^{-1}\right)^{m}$, i.e., $\left\langle\left(d g g^{-1}\right)^{m}, Z_{n}\right\rangle=\delta_{n}^{m}$. Again, by using some representation $\chi$ we have $Z_{m}=$ $\left(T_{m}\right)_{i j} g_{j k} \frac{\partial}{\partial g_{i k}}$ and hence the second relation in (B.9) reads $\left\{L_{m}, g_{i j}\right\}=-\left(T_{m} g\right)_{i j}$, implying that the left-currents generate the left translation. Of course, the two generators commute:

$$
\left\{R_{m}, L_{n}\right\}=0
$$

From (B.3) and (B.8) the Hamilton equations read

$$
\dot{g}=\{g, H\}=-g R, \quad \dot{R}=\{R, H\}=0,
$$

where dots denote differentiation with respect to time $t$. Combining the two equations in (B.11) we get

$$
\frac{d}{d t}\left(g^{-1} \dot{g}\right)=0
$$

To see that (B.12) in fact describes geodesic motion of a particle moving on the group manifold $\mathrm{G}$, let us first recall that a natural Riemannian metric on the group $\mathrm{G}$ is given by the formula

$$
d s^{2}=g_{\mu \nu} d x^{\mu} \otimes d x^{\nu}:=\operatorname{Tr}(v \otimes v),
$$

where $\left\{x^{\mu}\right\}, \mu=1, \ldots, N(N=\operatorname{dimG})$ is a coordinate on $\mathrm{G}$, and $v:=g^{-1} d g$ is the vielbein defined from the left-invariant 1-form. Expanding the vielbein $v=v^{m} T_{m}=$ $v_{\mu} d x^{\mu}=v_{\mu}^{m} d x^{\mu} T_{m}$, one finds that the metric in (B.13) reads $g_{\mu \nu}=\eta_{m n} v_{\mu}^{m} v_{\nu}^{n}$, where $\eta_{m n}$ is the flat metric (i.e., the metric in the vielbein frame) defined in Appendix A. Using the metric, it is now easy to rewrite (B.12) in the desired form

$$
\ddot{x}^{\mu}+\Gamma_{\nu \lambda}^{\mu} \dot{x}^{\nu} \dot{x}^{\lambda}=0
$$

with the Levi-Civita connection,

$$
\Gamma_{\nu \lambda}^{\mu}=\frac{1}{2} g^{\mu \rho}\left(\partial_{\nu} g_{\rho \lambda}+\partial_{\lambda} g_{\rho \nu}-\partial_{\rho} g_{\nu \lambda}\right) .
$$




\section{Appendix C. Metric on the coset $\mathrm{G} / \mathrm{H}$}

In this appendix we shall give a brief account of the metric on the coset space $\mathrm{G} / \mathrm{H}$ [39]. Our purpose here is to provide a simple procedure to determine the constant $c_{\chi}$, which allows us to evaluate explicitly the topological (Chern) numbers of the H-connections in the text.

To furnish a Riemannian metric on $\mathrm{G} / \mathrm{H}$ where $\mathrm{G}$ is a semisimple, compact group, we first note that, given a (local) section $\sigma: \mathrm{G} / \mathrm{H} \mapsto \mathrm{G}$ in $\mathrm{G}$, we can decompose $g \in \mathrm{G}$ as

$$
g=\sigma h, \quad \text { where } \quad \sigma \in \mathrm{G}, \quad h \in \mathrm{H} .
$$

Using the section, we consider the 1 -form $\sigma^{-1} d \sigma$ and its decomposition with respect to $\mathfrak{g}=\mathfrak{h} \oplus \mathfrak{r}$,

$$
\sigma^{-1} d \sigma=A+e, \quad \text { where } \quad A:=\left.\sigma^{-1} d \sigma\right|_{\mathfrak{h}}, \quad e:=\left.\sigma^{-1} d \sigma\right|_{\mathfrak{r}} .
$$

The 1-form $A$ is the $\mathrm{H}$-connection and is discussed in detail in the text, while the 1-form $e$ is regarded as a vielbein. As we did on the group manifold $\mathrm{G}$, we define a metric on the coset manifold $\mathrm{G} / \mathrm{H}$ by

$$
d s^{2}=g_{\alpha \beta} d q^{\alpha} \otimes d q^{\beta}:=\operatorname{Tr}(e \otimes e),
$$

where $\left\{q^{\alpha}\right\}, \alpha=1, \ldots, n(n=\operatorname{dim}(\mathrm{G} / \mathrm{H}))$, is a set of local coordinates on $\mathrm{G} / \mathrm{H}$. Expanding the vielbein $e=e^{a} T_{a}=e_{\alpha} d q^{\alpha}=e_{\alpha}^{a} d q^{\alpha} T_{a}$, one finds the metric $g_{\alpha \beta}=\eta_{a b} e_{\alpha}^{a} e_{\beta}^{b}$, where $\eta_{a b}:=\left(T_{a}, T_{b}\right)$ is the (flat) metric defined by the restriction of the metric $\eta_{m n}$ to the subspace $\mathfrak{r}$. Note that the left translation $g \rightarrow \tilde{g} g$ with $\tilde{g} \in \mathrm{G}$ induces a transformation $\sigma(q) \rightarrow \sigma(\tilde{g} q)=\tilde{g} \sigma(q) h_{\sigma}^{-1}(\tilde{g}, q)$ where $h_{\sigma} \in \mathrm{H}$ (see (2.9)), and hence the vielbein $e$ undergoes the $\mathrm{SO}(n)$ transformation $e \rightarrow h_{\sigma} e h_{\sigma}^{-1}$ in the vielbein frame, leaving the metric $g_{\alpha \beta}$ invariant. Once we have a vielbein (or metric) on $\mathrm{G} / \mathrm{H}$ the volume form is defined as

$$
\Omega=\sqrt{\operatorname{det} \eta_{a b}} e^{1} \wedge e^{2} \wedge \cdots \wedge e^{n}=\sqrt{\operatorname{det} g_{\alpha \beta}} d q^{1} \wedge d q^{2} \wedge \cdots \wedge d q^{n} .
$$

Let us specialize to the case $\mathrm{G} / \mathrm{H}=\mathrm{SO}(n+1) / \mathrm{SO}(n) \simeq S^{n}$. Working in the defining representation $\chi_{\text {def }}$ of $\operatorname{so}(n+1)$, we shall choose a basis $\left\{T_{j k}\right\}, j, k=1, \ldots, n+1$, by

$$
\left(T_{j k}\right)_{l m}:=\delta_{j l} \delta_{k m}-\delta_{j m} \delta_{k l},
$$


which has the trace,

$$
\operatorname{tr}\left(T_{j k} T_{j^{\prime} k^{\prime}}\right)=-2 \delta_{j j^{\prime}} \delta_{k k^{\prime}}
$$

Defining $T_{a}=T_{a, n+1}$ for $a=1, \ldots, n$, we have the orthogonal decomposition

$$
\mathfrak{g}=\mathfrak{h} \oplus \mathfrak{r}=\operatorname{span}\left\{T_{a b}\right\} \oplus \operatorname{span}\left\{T_{a}\right\}, \quad a, b=1, \ldots, n
$$

If we choose for the decomposition (C.1) the section $\sigma=e^{\theta^{a} T_{a}}$, we find that

$$
\sigma=\left(\begin{array}{cc}
\cos \sqrt{\theta \theta^{t}} & \frac{\sin |\theta|}{|\theta|} \theta \\
-\frac{\sin |\theta|}{|\theta|} \theta^{t} & \cos |\theta|
\end{array}\right),
$$

where we have used the column vector $\theta$ with $\theta^{t}=\left(\theta^{1}, \ldots, \theta^{n}\right)$ and $|\theta|=\sqrt{\theta^{t} \theta}=$ $\sqrt{\sum_{a}\left(\theta^{a}\right)^{2}}$. Then we find that the new coordinate $q=\left(q^{1}, \ldots, q^{n+1}\right)$ with

$$
q^{a}:=\theta^{a} \frac{\sin |\theta|}{|\theta|}, \quad a=1, \ldots, n, \quad q^{n+1}:=\cos |\theta|,
$$

satisfies the condition $\sum_{a=1}^{n+1}\left(q^{a}\right)^{2}=1$, and thus it serves as a coordinate on $S^{n}$ with unit radius.

The metric on the coset is given by (C.3), but in order to specify the radius $r$ of $S^{n}$ to be unity for the coset space we must determine the constant $c_{\chi}$ used in the innerproduct appropriately. A practical way for this is the following. Take the two points, the north pole $q_{N}$ and the south pole $q_{S}$ on $S^{n}$. In terms of the coordinate $q$ these points may be taken to be

$$
q_{N}=(0,0, \ldots, 0,1), \quad q_{S}=(0,0, \ldots, 0,-1) .
$$

From (C.9) a great circle connecting the two points is given by $\theta^{1}(t)=\pi t, \theta^{a}(t)=0$ for $a \neq 1(0 \leq t \leq 1)$; indeed we have $q(t):=q(\theta(t))=(\sin \pi t, 0, \ldots, 0, \cos \pi t)$ and hence $q(0)=q_{N}, q(1)=q_{S}$. Accordingly, we get $\sigma(q(t))=e^{\pi t T_{1}}$ and thus the vielbein is just $e=\pi d t T_{1}$. Since the distance between the two points is evaluated from (C.3) as

$$
d\left(q_{N}, q_{S}\right):=\int_{q_{N}}^{q_{S}} d s=\pi \sqrt{-c_{\chi_{\operatorname{def}}}^{-1} \operatorname{tr} T_{1}^{2}} \int_{0}^{1} d t
$$

and since this must be $\pi$ for $S^{n}$ with unit radius, we deduce that $c_{\chi_{\text {def }}}=2$ for the defining representation on account of the normalization (C.6). 
Let us specialize further to the $n=2 m$ situation; $\mathrm{G} / \mathrm{H}=\mathrm{SO}(2 m+1) / \mathrm{SO}(2 m) \simeq S^{2 m}$. In this case, from the indices of the representations, i.e., from the fact that the quadratic trace in the adjoint (spinor) representation of $\mathrm{so}(2 m+1)$ is $(2 m-1)$ times $\left(2^{m-3}\right.$ times) that of the defining representation, we find

$$
c_{\chi_{\mathrm{def}}}=2, \quad c_{\chi_{\mathrm{ad}}}=2(2 m-1), \quad c_{\chi_{\mathrm{sp}}}=2^{m-2} .
$$

If we wish to have a generic radius $r$, we just rescale those $c_{\chi}$ by $\frac{1}{r^{2}} ;$ e.g., we have $c_{\chi_{\text {def }}}=\frac{2}{r^{2}}$. 


\section{Appendix D. H-connection as a Yang-Mills connection on $\mathrm{G} / \mathrm{H}$}

We wish to show that, for a semisimple, compact $\mathrm{G}$, the $\mathrm{H}$-connection $A$ is in fact a solution of the Yang-Mills equation on the coset space $\mathrm{G} / \mathrm{H}$, that is, the curvature $F:=d A+A \wedge A$ satisfies

$$
D^{*} F=0,
$$

where $D^{*}:=-* D *$ is the adjoint operator of $D$. The proof is straightforward:

Proof. From the definition of the Hodge-star $*$,

$$
*\left(e^{a_{1}} \wedge \cdots \wedge e^{a_{p}}\right)=\frac{1}{(n-p) !} \varepsilon^{a_{1} \ldots a_{p}}{ }_{a_{p+1} \ldots a_{n}} e^{a_{p+1}} \wedge \cdots \wedge e^{a_{n}},
$$

we have for $D * F=d * F+[A, * F]$,

$$
\begin{aligned}
(D * F)^{i}=-\frac{1}{(n-3) ! 2} f_{a b}^{i} \varepsilon^{a b}{ }_{a_{3} \ldots a_{n}} d\left(e^{a_{3}}\right) \wedge e^{a_{4}} \wedge \cdots \wedge e^{a_{n}} & \\
& -\frac{1}{(n-2) ! 2} f_{j k}^{i} f_{a b}^{k} \varepsilon^{a b}{ }_{a_{3} \ldots a_{n}} A^{j} \wedge e^{a_{3}} \wedge \cdots \wedge e^{a_{n}} .
\end{aligned}
$$

Using the Maurer-Cartan equation $D e=-\left.e \wedge e\right|_{\mathfrak{r}}$ in (6.5) the first term in (D.3) becomes

$$
d * F^{i}=\frac{1}{(n-3) ! 4} f_{a b}^{i}\left(f_{c d}^{a_{3}}+2 f_{j d}^{a_{3}} A_{c}^{j}\right) \varepsilon^{a b}{ }_{a_{3} \ldots a_{n}} e^{c} \wedge e^{d} \wedge e^{a_{4}} \wedge \cdots \wedge e^{a_{n}},
$$

where we have expanded $A^{j}=A_{c}^{j} e^{c}$ in the vielbein frame. Then applying $*$ we get

$$
\begin{aligned}
* d * F^{i} & =\frac{1}{(n-3) ! 4} f_{a b}^{i}\left(f_{c d}^{a_{3}}+2 f_{j d}^{a_{3}} A_{c}^{j}\right) \varepsilon_{a_{3} \ldots a_{n}}^{a b} \varepsilon^{c d a_{4} \ldots a_{n}} e^{e} \\
& =\frac{1}{2}(-1)^{n-1} f_{a b}^{i} f_{a b}^{e} e^{e}+(-1)^{n-1}\left(f_{e j}^{b} f_{b i}^{a}+f_{i e}^{b} f_{b j}^{a}\right) A_{a}^{j} e^{e},
\end{aligned}
$$

where we have used a basis in which the metric in the vielbein frame takes the form $\eta_{m n}=\delta_{m n}$, which is possible because of the compactness of the group G (see Appendix A). On the other hand, applying $*$ to the second term in (D.3) we obtain

$$
*[A, * F]^{i}=-\frac{1}{(n-2) ! 2} f_{j k}^{i} f_{a b}^{k} A_{c}^{j} \varepsilon_{a_{3} \ldots a_{n}}^{a b} \varepsilon^{c a_{3} \ldots a_{n}} e^{e}=(-1)^{n-1} f_{j k}^{i} f_{a e}^{k} A_{a}^{j} e^{e} .
$$

Combining (D.5) and (D.6) we find

$$
(-1)^{n}\left(D^{*} F\right)^{i}=\frac{1}{2} f_{a b}^{i} f_{a b}^{e} e^{e}+\left(f_{e j}^{b} f_{b i}^{a}+f_{i e}^{b} f_{b j}^{a}+f_{j k}^{i} f_{a e}^{k}\right) A_{a}^{j} e^{e} .
$$

By using the Jacobi identity and the orthogonality of the decomposition (A.4),

$$
f_{a b}^{j} f_{j i}^{k}+f_{i a}^{c} f_{c b}^{k}+f_{b i}^{c} f_{c a}^{k}=0, \quad \text { and } \quad f_{e a}^{b} f_{i b}^{a}=\operatorname{tr}\left(\pi^{\chi_{\text {ad }}}\left(T_{e}\right) \pi^{\chi_{\text {ad }}}\left(T_{i}\right)\right)=0,
$$

we obtain the Yang-Mills equation (D.1). Q.E.D. 


\section{References}

[1] P.A.M. Dirac, "Lectures on Quantum Mechanics" (Yeshiva, New York, 1964).

[2] R. Jackiw, in "Relativity, Groups and Topology II" eds. B.S. DeWitt and R. Stora (North-Holland, Amsterdam, 1984).

[3] K.V. Kuchař, Phys. Rev. D35 (1987) 596.

[4] D. McMullan and J. Paterson, J. Math. Phys. 30 (1989) 477, 487.

[5] I. Singer, Commun. Math. Phys. 60 (1978) 7.

[6] G.W. Mackey, "Induced Representations of Groups and Quantum Mechanics" (Benjamin, New York, 1969).

[7] See, for example, N. Woodhouse, "Geometric Quantization" (OUP, Oxford, 1980).

[8] C.J. Isham, in "Relativity, Groups and Topology II" eds. B.S. DeWitt and R. Stora (North-Holland, Amsterdam, 1984).

[9] N.P. Landsman, Rev. Math. Phys. 2 (1990) 45, 73.

[10] N.P. Landsman and N. Linden, Nucl. Phys. B365 (1991) 121.

[11] D. McMullan and I. Tsutsui, "Functional gauge structures in gauge theories", in preparation.

[12] L. Faddeev and S. Shatashviti, Phys. Lett. 167B (1986) 225.

[13] H.B. Nielsen and D. Rohrlich, Nucl. Phys. B299 (1988) 471.

[14] V.S. Varadarajan, "Geometry of Quantum Theory" vol II (Van Nostrand, New York, 1970).

[15] M. Koch, "Quantum mechanics on homogeneous configuration spaces and classical particles in Yang-Mills fields", Universität Freiburg preprint THEP 91/19.

[16] N.P. Landsman, Rev. Math. Phys. 4 (1992) 503.

[17] Y. Ohnuki and S. Kitakado, Mod. Phys. Lett. A7 (1992) 2477; J. Math. Phys. 34 (1993) 2827.

[18] S. Tanimura, "Quantum mechanics on manifolds", Nagoya Univ. preprint DPNU-93-21. 
[19] A.M. Perelomov, "Integrable Systems of Classical mechanics and Lie Algebras" (Birkhäuser Verlag, Basel, 1990).

[20] L.S. Schulman, "Techniques and Applications of Path Integration" (Wiely, New York, 1981); L. Schulman, Phys. Rev. 176 (1968) 1558;

J.F. Hamilton and L.S. Schulman, J. Math. Phys. 12 (1971) 161.

[21] A.O. Barut and R. Raczka, "Theory of Group Representations and Applications" (Polish Scientific Publishers, Warszawa, 1977).

[22] L.D. Faddeev, Theor. Math. Phys. 1 (1970) 1;

P. Senjanovic, Ann. Phys. 100 (1976) 227.

[23] D. McMullan, "Constrained quantization, gauge fixing and the Gribov ambiguity", DIAS-STP-92-23 to appear in Commun. Math. Phys.

[24] A. Alekseev, L. Faddeev and S. Shatashvili, J. Geom. Phys. 5 (1989) 391.

[25] R. Abraham and J.E. Marsden, "Foundations of Machanics" Second Edition, (Benjamin/Cummings, Reading, 1978).

[26] S.E. Humphreys, "Introduction to Lie Algebras and Representation Theory" (Springer-Verlag, Berlin, 1972).

[27] S.K. Wong, Nuovo Cimento 65A (1970) 689.

[28] Y.-S. Wu and A. Zee, Nucl. Phys. B258 (1985) 157.

[29] A.P. Balachandran, G. Marmo, B.-S. Skagerstam and A. Stern, "Gauge Symmetries and Fibre Bundles: Application to Particle Dynamics", Lecture Notes in Physics 188 (Springer-Verlag, Berlin, 1983).

[30] F.A. Bais and P. Batenburg, Nucl. Phys. B269 (1986) 363.

[31] H.T. Laquer, in "Group actions on manifolds", Contemp. Math. 36 (AMS, Providence, 1983).

[32] I. Tsutsui and L. Fehér, Phys. Lett. 294B (1992) 209.

[33] N.P. Landsman and N. Linden, Nucl. Phys. B371 (1992) 415.

[34] P. Nelson and L. Alveraz-Gaumé, Commun. Math. Phys. 99 (1985) 103.

[35] Y. Aharonov and D. Bohm, Phys. Rev. 115 (1959) 485.

[36] M.V. Berry, Proc. R. Lond. A392 (1984) 45. 
[37] F. Wilczek and A. Zee, Phys. Rev. Lett. 52 (1984) 2111.

[38] H. Kuratsuji, Phys. Rev. Lett. 61 (1988) 1687; Int. J. Mod. Phys. A7 (1992) 4595;

H. Kuratsuji and K. Takada, Mod. Phys. Lett. A5 (1990) 1765.

[39] R. Gilmore, "Lie Groups, Lie Algebras and Some of Their Applications" (Willey, New York, 1974). 